\title{
Large-area display textiles integrated with functional systems
}

Huisheng Peng ( $\square$ penghs@fudan.edu.cn )

Fudan University https://orcid.org/0000-0002-2142-2945

\section{Xiang Shi}

Fudan University

\section{Yong Zuo}

Fudan University

\section{Peng Zhai}

Fudan University

Jiahao Shen

Fudan University

\section{Yangyiwei Yang}

Technische Universität Darmstadt

\section{Zhen Gao}

Fudan University

Meng Liao

Fudan University

Jiawei Wang

Fudan University

\section{Xiaojie Xu}

Fudan University

\section{Qi Tong}

Fudan University

\section{Bo Zhang}

Fudan University https://orcid.org/0000-0002-7853-979X

\section{Xuemei Sun}

State Key Laboratory of Molecular Engineering of Polymers, Department of Macromolecular Science and Laboratory of Advanced Materials, and Department of Chemistry, Fudan University

https://orcid.org/0000-0002-2583-8593

\section{Lihua Zhang}

Fudan University

\section{Qibing Pei}

University of California, Los Angeles https://orcid.org/0000-0003-1669-1734

Dayong Jin 
University of Technology Sydney https://orcid.org/0000-0003-1046-2666

\section{Peining Chen}

Fudan University

\section{Research Article}

Keywords: textiles, electronic devices, fabrication

Posted Date: August 17th, 2020

DOI: https://doi.org/10.21203/rs.3.rs-56463/v1

License: (c) (1) This work is licensed under a Creative Commons Attribution 4.0 International License. Read Full License

Version of Record: A version of this preprint was published at Nature on March 10th, 2021. See the published version at https://doi.org/10.1038/s41586-021-03295-8. 
3 Xiang $\mathrm{Shi}^{1 \dagger}$, Yong Zuo ${ }^{1 \dagger}$, Peng Zhai ${ }^{2 \dagger}$, Jiahao Shen ${ }^{3}$, Yangyiwei Yang ${ }^{4}$, Zhen Gao ${ }^{1}$, Meng Liao ${ }^{1}$,

4 Jiawei Wang ${ }^{1}$, Xiaojie Xu${ }^{1}$, Qi Tong ${ }^{3}$, Bo Zhang ${ }^{1}$, Xuemei Sun ${ }^{1}$, Lihua Zhang ${ }^{2}$, Qibing Pei ${ }^{5}$,

5 Dayong Jin ${ }^{6}$, Peining Chen ${ }^{1 *}$, Huisheng Peng ${ }^{1 *}$

$6 \quad{ }^{1}$ State Key Laboratory of Molecular Engineering of Polymers, Department of Macromolecular

7 Science and Laboratory of Advanced Materials, Fudan University, Shanghai, 200438, China.

$8 \quad{ }^{2}$ The Institute of AI and Robotics, Fudan University, Shanghai, 200433, China.

$9 \quad{ }^{3}$ Department of Aeronautics and Astronautics, Fudan University, Shanghai, 200433, China.

$10{ }^{4}$ Mechanics of Functional Materials Division, Institute of Materials Science, Technische

11 Universität Darmstadt, Darmstadt, 64287, Germany.

$12{ }^{5}$ Department of Materials Science and Engineering, Henry Samueli School of Engineering and

13 Applied Science, University of California Los Angeles, Los Angeles, CA, 90095, USA.

$14{ }^{6}$ Institute for Biomedical Materials \& Devices (IBMD), School of Mathematical and Physical

15 Sciences, Faculty of Science, University of Technology Sydney, NSW, 2007, Australia.

17 Displays are basic building blocks of modern electronics ${ }^{1,2}$. Integrating displays into textiles 18 offers exciting opportunities for smart electronic textiles - the ultimate form of wearables 19 poised to change the way we interact with electronic devices ${ }^{3-6}$. Display textiles serve to 20 bridge human-machine interactions ${ }^{7-9}$, offering for instance, a real-time communication tool

21 for individuals with voice or speech disorders. Electronic textiles capable of communicating ${ }^{10}$, 22 sensing ${ }^{11,12}$ and supplying electricity ${ }^{13,14}$ have been reported previously. However, textiles 23 with functional, large-area displays have not been achieved so far because obtaining small 24 illuminating units that are both durable and easy to assemble over a wide area is challenging. 25 Here, we report a $6 \mathrm{~m}(\mathrm{~L}) \times 25 \mathrm{~cm}(\mathrm{~W})$ display textile containing $5 \times 10^{5}$ electroluminescent 26 (EL) units narrowly spaced to $\sim 800 \mu \mathrm{m}$. Weaving conductive weft and luminescent warp 27 fibres forms micron-scale EL units at the weft-warp contact points. Brightness between EL 28 units deviates by $<6.3 \%$ and remains stable even when the textile is bent, stretched or pressed. 29 We attribute this uniform and stable lighting to the smooth luminescent coating around the 
warp fibres and homogenous electric field distribution at the contact points. Our display textile is flexible and breathable and withstands repeatable machine-washing, making them suitable for practical applications. We show an integrated textile system consisting of display, keyboard and power supply can serve as a communication tool, which could potentially drive the Internet of Things in various areas including healthcare. Our approach unifies the fabrication and function of electronic devices with textiles, and we expect weaving fibre materials to shape the next-generation electronics.

Display devices have evolved from rigid panels to flexible thin films ${ }^{15}$. However, the configuration and fabrication of electronic textiles are different from conventional film devices such as organic light-emitting diodes (OLEDs) that are currently used to construct flexible displays. Textiles are woven from fibres, forming rough and porous structures that can deform and fit the contours of the human body ${ }^{16,17}$. OLEDs, on the other hand, are made by depositing multiple layers of semiconducting organic thin films between cathode and anode electrodes that are placed on planar substrates such as glass or plastic ${ }^{18}$. Depositing organic thin films on fibres that are suitable for weaving into flexible display textiles is very difficult because these thin films are too fragile to withstand the chafing during weaving. The evaporation method used to make OLEDs is not amenable to large-scale fabrication of fibre electrodes. More importantly, because light emission in OLEDs depends on carrier injection and transport between the anode and cathode ${ }^{19,20}$, weaving warps and wefts cannot provide sufficient high quality ohmic contact between the electrodes and semiconducting layers for illumination.

In our study, we used electric-field driven devices based on $\mathrm{ZnS}$ phosphor to weave a display textile. Unlike OLED devices, $\mathrm{ZnS}$ phosphor dispersed in an insulating polymer matrix is activated by alternating electric field across polymer matrix ${ }^{21}$. Such electric-field driven devices require only spatial contacts between wefts and warps to illuminate ${ }^{22,23}$, making them intrinsically durable and suitable for large-scale production. We prepared transparent (over 90\% transmittance) conductive weft fibres by melt-spinning ionic-liquid-doped polyurethane gel (Extended Data Fig. 1a-c), and luminescent warp fibres by coating commercially available $\mathrm{ZnS}$ phosphor on silver-plated conductive yarn (Extended Data Fig. 1d-f). This solution-based coating presents a simple process

60 to obtain luminescent warp fibres continuously. We chose polyurethane as polymer matrix because

61 it is durable to friction, compression and bending during weaving. To ensure uniform coating of 
$62 \mathrm{ZnS}$, we dip-coated the conductive yarn in $\mathrm{ZnS}$ phosphor slurry and passed it through a home63 made scraping micro-pinhole before drying (Extended Data Fig. 2a). The micro-pinhole 64 smoothed the coating along the longitudinal and circumferential directions (Extended Data Fig.

$65 \mathbf{2 b}, \mathbf{c})$. Different diameters of the micro-pinhole were used to tune the thickness of the $\mathrm{ZnS}$ 66 phosphor layer. We used an optimized thickness of $\sim 70 \mu \mathrm{m}$ in our experiments unless specified 67 otherwise. To evaluate the uniformity of the luminescent coating, we placed a 100-metre-long 68 luminescent warp into salt water and applied alternating voltage between them (Extended Data 69 Fig. 2d). The luminescence remained stable even when twisted (Extended Data Fig. 2e). For a 70 30-metre-long fibre, the luminescence intensity varied by less than 10\% (Extended Data Fig. 2f, 71 g). The intensity along the circumference at different locations of the fibre was almost identical 72 and was independent of observation angle (Extended Data Fig. 2h). Fibres with an uneven ZnS 73 phosphor coating (Extended Data Fig. 2i) showed uneven brightness and failure in some EL units 74 (Extended Data Fig. 2j, k), indicating that light emission requires a uniform luminescent coating.

When the conductive weft and luminescent warp fibres are woven with cotton yarn using an industrial rapier loom, each interlaced weft and warp forms an effective EL unit (Fig. 1a, Extended Data Fig. 1h, i). Using this method, we produced a $6 \mathrm{~m}(\mathrm{~L}) \times 25 \mathrm{~cm}(\mathrm{~W})$ large-area display textile containing approximately $5 \times 10^{5}$ EL units (Fig. 1b and Supplementary Movie 1). The relative deviations of emission intensity for $600 \mathrm{EL}$ units examined were narrowly between $-6.3 \%$ to $5.2 \%$ (Fig. 1c). Such small differences $(<10 \%)$ in intensity indicate that these fibres are well-suited for making large-area display textiles at scale (Fig. 1d). Even after 1,000 cycles of bending (Fig. 1e), stretching (Fig. 1f) and pressing (Fig. 1g), the intensity of these 600 EL units remained stable (with $<10 \%$ variations). We also obtained colourful textiles (Fig. 1h) with uniformly spaced EL units (Fig. 1i) by doping different elements such as copper and manganese into the $\mathrm{ZnS}$ phosphor ${ }^{24}$. Because the fibres are woven, the density of the EL units can be easily tuned by adjusting the weaving parameters to change the distances of the weft-warp contact points (Fig. 1j). The narrowest spacing we achieved here is approximately $800 \mu \mathrm{m}$.

To turn on the EL units, we applied alternating voltage to the luminescent warps and conductive 91 wefts, generating a low microampere current to power the units (Fig. 2a). Electric-field-induced 92 excitation of the luminescent centre and recombination of electron-hole pairs ${ }^{25}$ result in light 93 emission from the $\mathrm{ZnS}$ phosphor at the weft-warp contact area. By varying the applied electricity, 
94 we could accurately tune the luminance of the EL unit. A luminance of $115.1 \mathrm{~cd} / \mathrm{m}^{2}$ was achieved 95 at current density of $1.8 \mathrm{~mA} / \mathrm{cm}^{2}$ and power consumption of $363.1 \mu \mathrm{W}$ (Extended Data Fig. 3a96 d). At such a low power consumption, heating was negligible (Extended Data Fig. 3e, f), which 97 is crucial for large-area clothing applications.

99 Because light emission also depends on how uniform the electric field is at the curved contact area 100 between the luminescent warp and conductive weft, we used finite element method to simulate the 101 electric field distribution in the luminescent layer (Fig. 2b). We found that the distribution at the 102 curved contact under applied voltage was as uniform as a planar EL device (Fig. 2b, Extended 103 Data Fig. 4a-f) and remained uniform even when the contact area was changed (Fig. 2c, Extended 104 Data Fig. 4g). We attribute this electric field homogeneity to the elastic conductive weft that 105 readily deforms to fit the curved and less elastic surface of the luminescent warp (Extended Data 106 Fig. 1g, h). Light emission occurred even when the conductive weft leaned, twisted and knotted 107 with the luminescent warp (Fig. 2d, Supplementary Movie 2). EL mapping images show that EL 108 intensities and unit areas remained nearly unchanged when the transparent conductive weft was 109 moved along the luminescent warp (Fig. 2e), rotated around the contacting point (Fig. 2f), and 110 bent with increasing bending angles (Fig. 2g). As the conductive weft slid along the luminescent 111 warp at increments of $0.5 \mathrm{~mm}$, the luminance varied by less than $2.2 \%$ for a distance up to $3 \mathrm{~mm}$ 112 (Fig. 2h). When the transparent weft rotated by $\pm 15^{\circ}$ from the position perpendicular to the 113 luminescent warp, the EL intensity fluctuated by less than $2.6 \%$ (Fig. 2i). Furthermore, due to the 114 elasticity of the transparent weft, luminescence recovered instantly and remained stable over 100 115 cycles of pressing and releasing of the EL unit (Fig. 2j). Bending the transparent weft or 116 luminescent warp up to $1.8 \mathrm{~mm}$ from its original state also resulted in fluctuations of less than $2.3 \%$ 117 (Fig. 2k, l). Because the fibre is cylindrical, the EL intensity was well-maintained when the 118 transparent weft was rolled around its central axis (Extended Data Fig. 5). The inert and non119 volatile nature of the ionic liquid $^{26}$ in the transparent conductive weft also contributed to the 120 electrical and optical stability of the EL unit (Extended Data Fig. 6a, b). Leaving the textile in 121 the open air for 1 month did not show any obvious decrease in luminance (Extended Data Fig. 122 6c). When we coated the wefts and warps with a thin silicone protective layer, the brightness of 123 the EL units endured repeated machine wash cycles (Extended Data Fig. 6d-f).

125 To show our weaving strategy is general, we used it to produce other electronic functions within 
126 the textile (e.g., keyboard and power supply). To create a textile keyboard that functions through 127 dynamic contact, we wove low resistance warp (silver-plated yarn) with high resistance weft 128 (carbon fibre) to form a $4 \times 4$ keyboard (Extended Data Fig. 7a), where the intersections of the 129 weft and warp form the keypresses (Extended Data Fig. 7b, c). Pressing the keypress turns it on 130 while releasing turns it off (Extended Data Fig. 7d, e). The keyboard works by reading the voltage 131 between the metallic and carbon fibres (sample voltage, $\mathrm{V}_{\mathrm{s}}$ ) under an applied voltage $\left(\mathrm{V}_{\mathrm{cc}}\right)$ of $5 \mathrm{~V}$.

132 Each keypress in the $4 \times 4$ keyboard is distinguished by the different sample voltage recorded when 133 the keypress is pressed (Extended Data Fig. 7f). As power supply, we wove photoanode wefts 134 with silver-plated conductive yarns to harvest solar energy (Extended Data Fig. 8a-f). The 135 photoanode weft is a titanium (Ti) wire coated with a photoactive layer composed of titanium 136 dioxide $\left(\mathrm{TiO}_{2}\right)$ nanotubes as the electron transport layer, dye molecules as sensitizer and copper 137 iodide $(\mathrm{CuI})$ as the solid electrolyte. Integrating these warps and wefts with battery fibres 138 assembled from flexible $\mathrm{MnO}_{2}$-coated carbon nanotube fibre (cathode), zinc wire (anode) and $139 \mathrm{ZnSO}_{4}$ gel electrolyte, we realised both power generation and storage in the textile (Extended 140 Data Fig. 8g-i). With display, keyboard and power supply, we can design a variety of multi141 functional integrated textile systems for different applications (Fig. 3a, Extended Data Fig. 9).

143 As a proof-of-concept, we connected the woven display, keyboard and power supply to a display 144 driver, microcontroller and Bluetooth module (Fig. 3b) and used the integrated textile system as 145 an interactive navigation display (Fig. 3c). Through the Bluetooth module, real time location on a 146 T-junction from a smartphone was transferred to the textile (Fig. 3d). To output the image on the 147 display textile, electrical signals from the driver circuit are scanned row by row onto the array of 148 EL units (Fig. 3e, Supplementary Movie 3). Our integrated textile system can also function as a 149 communication tool, where information is input and displayed on the textile (Fig. 3f, 150 Supplementary Movie 3). We demonstrate this using numbers '1', '2', and ' 3 '. Each number is 151 assigned a keypress and the microcontroller is programmed to output the number when the 152 corresponding keypress is pushed (Fig. 3g). With the Bluetooth module, messages can also be sent, 153 received and displayed between our integrated textile system and a smartphone (Fig. 3h).

155 To demonstrate the potential of display textiles in healthcare, we collected electroencephalogram 156 signals from volunteers playing a race car game and those who were meditating. Brain waves in 157 relaxed volunteers were mostly low frequency (Fig. 3i) while those in anxious volunteers were 
158 mostly high frequency ${ }^{27}$ (Fig. 3j). We processed the signals on a computer and sent words 159 corresponding to the mental state of the respective volunteers to the microcontroller through the 160 Bluetooth module for display. In the future, together with ways to decode complicated brain waves, 161 we envision display textiles like ours to become effective communication tools for individuals 162 with voice, speech or language disorders ${ }^{28}$ (Fig. 3k). Such a tool will enable these individuals to 163 express themselves and reconnect with the world around them (Fig. 31).

165 In summary, we present a functional, large-area display textile by weaving conductive and 166 luminescent fibres with cotton yarn to form EL units directly within the textile. Our method is 167 simple and can be used to weave other electronic functions such as a keyboard and power supply 168 into the textile to form a multi-functional integrated textile system for various applications. 169 Because of the network of wefts and warps, each EL unit in our display textile can be uniquely 170 identified and lit in a programmable way using a driver circuit. We show such an electronic textile 171 can be useful communication tools. With the integration of more functionalities, we expect these 172 smart textiles to form the communication tools of the future. 
1. Larson, C. et al. Highly stretchable electroluminescent skin for optical signaling and tactile sensing. Science 351, 1071-1074 (2016).

2. Tan, Y. J. et al. A transparent, self-healing and high- $\kappa$ dielectric for low-field-emission stretchable optoelectronics. Nat. Mater. 19, 182-188 (2020).

3. Tian, X. et al. Wireless body sensor networks based on metamaterial textiles. Nat. Electron. 2 , 243-251 (2019).

4. Chen, G. R., Li, Y. Z., Bick, M. \& Chen, J. Smart textiles for electricity generation. Chem. Rev. 120, 3668-3720 (2020).

5. Weng, W., Chen, P. N., He, S. S., Sun, X. M. \& Peng, H. S. Smart electronic textiles. Angew. Chem. Int. Ed. 55, 6140-6169 (2016).

6. Carey, T. et al. Fully inkjet-printed two-dimensional material field-effect heterojunctions for wearable and textile electronics. Nat. Commun. 8, 1202 (2017).

7. Kim, J. et al. Ultrathin quantum dot display integrated with wearable electronics. Adv. Mater. 29, 1700217 (2017).

8. Son, D. et al. An integrated self-healable electronic skin system fabricated via dynamic reconstruction of a nanostructured conducting network. Nat. Nanotechnol. 13, 1057-1065 (2018).

9. Yin, D. et al. Efficient and mechanically robust stretchable organic light-emitting devices by a laser-programmable buckling process. Nat. Commun. 7, 11573 (2016).

10. Rein, M. et al. Diode fibres for fabric-based optical communications. Nature 560, 214-218 (2018).

11. Leber, A. et al. Soft and stretchable liquid metal transmission lines as distributed probes of multimodal deformations. Nat. Electron. 3, 316-326 (2020).

12. Yang, A. N. et al. Fabric organic electrochemical transistors for biosensors. Adv. Mater. $\mathbf{3 0}$, 1800051 (2018).

13. Hatamvand, M. et al. Recent advances in fiber-shaped and planar-shaped textile solar cells. Nano Energy 71, 104609 (2020).

14. Jinno, H. et al. Stretchable and waterproof elastomer-coated organic photovoltaics for washable electronic textile applications. Nat. Energy 2, 780-785 (2017).

15. Koo, J. H., Kim, D. C., Shim, H. J., Kim, T. H. \& Kim, D. H. Flexible and stretchable smart display: materials, fabrication, device design, and system integration. Adv. Funct. Mater. 28, 
1801834 (2018).

16. de Mulatier, S., Nasreldin, M., Delattre, R., Ramuz, M. \& Djenizian, T. Electronic circuits integration in textiles for data processing in wearable technologies. Adv. Mater. Technol. 3, 1700320 (2018).

17. Wang, B. H. \& Facchetti, A. Mechanically flexible conductors for stretchable and wearable eskin and e-textile devices. Adv. Mater. 31, 1901408 (2019).

18. Prieto-Ruiz, J. P. et al. Enhancing light emission in interface engineered spin-OLEDs through spin-polarized injection at high voltages. Adv. Mater. 31, 1806817 (2019).

19. Fukagawa, H. et al. Long-lived flexible displays employing efficient and stable inverted organic light-emitting diodes. Adv. Mater. 30, 1706768 (2018).

20. Conaghan, P. J. et al. Highly efficient blue organic light-emitting diodes based on carbenemetal-amides. Nat. Commun. 11, 1758 (2020).

21. Zhou Y. et al. Bright stretchable electroluminescent devices based on silver nanowire electrodes and high-k thermoplastic elastomers. ACS Appl. Mater. Interfaces 10, 44760-44767 (2018).

23. Zhang, Z. T. et al. A stretchable and sensitive light-emitting fabric. J. Mater. Chem. C 5, 41394144 (2017).

24. Yang, C. H., Chen, B. H., Zhou, J. X., Chen, Y. M. \& Suo, Z. G. Electroluminescence of giant stretchability. Adv. Mater. 28, 4480-4484 (2016).

25. Chen, F. \& Xiang, Y. AC Powder Electroluminescence in Luminescent Materials and Applications (John Wiley \& Sons, Chichester, 2008).

26. Jin, M. L. et al. An ultrastable ionic chemiresistor skin with an intrinsically stretchable polymer electrolyte. Adv. Mater. 30, 1706851 (2018).

27. Müller-Putz, G. R., Riedl, R. \& Wriessnegger, S. C. Electroencephalography (EEG) as a research tool in the information systems discipline: foundations, measurement, and applications. Commun. Assoc. Inf. Syst. 37, 912-948 (2015).

28. Voice, speech and language research, https://www.nidcd.nih.gov/about/strategic-plan/20172021/voice-speech-and-language-research (2017). 


\section{Preparation of the transparent conductive weft}

240 Polyurethane ionic gel fibre was spun from the transparent ionic-liquid-doped polyurethane gel.

241 Thermoplastic polyurethane (TPU) (Covestro, Desmopan ${ }^{\circledR} 2786$ A) was firstly dissolved in N, N-

242 dimethylformamide (DMF) (Sinopharm) with a weight ratio of $1 / 4$ under mechanically stirring at

$24380{ }^{\circ} \mathrm{C}$ for $2 \mathrm{~h}$. Subsequently, 1-ethyl-3-methylimidazolium:bis (trifluoromethylsulfoyl) imide

244 ([EMIM $]^{+}\left[\mathrm{TFSI}^{-}\right)$ionic liquid (Aladdin) was added to the above TPU-DMF solution for further

245 stirring $\left(80^{\circ} \mathrm{C}\right.$ for $\left.1 \mathrm{~h}\right)$. The ionic gel flake was obtained by totally removing the solvent of DMF

246 in an oven box at $80{ }^{\circ} \mathrm{C}$ for $12 \mathrm{~h}$. Then melt-spinning was carried out using 3D printing system

247 (3D Bio-Architect ${ }^{\circledR}$ work station, Regenovo) with $0.25 \mathrm{~mm}$ inner diameter nozzle. The transparent

248 conductive weft was extruded at a melting zone temperature of $180{ }^{\circ} \mathrm{C}$ and cooled at room

249 temperature. To achieve water resistance, silicone protective layer (Dow Corning, 1-2577) could

250 be further dip-coated on the transparent conductive wefts.

\section{Preparation of the luminescent warp}

252 Commercially available ZnS phosphors (Shanghai Keyan Phosphor Technology Co., Ltd.) were 253 dispersed in waterborne polyurethane (U-9, Shanghai Sisheng Polymer Materials Co., Ltd.) with 254 a weight ratio of $3 / 1$ by mechanical stirring for $20 \mathrm{~min}$. After degassing in a vacuum oven, the as255 prepared mixtures were loaded on the silver-plated nylon yarns (100D, Hengtong X-silver 256 Speciality Textile Co., Ltd.) on a continuous producing line. Silver-plated yarns were dipped into 257 the $\mathrm{ZnS}$ phosphor dispersions and passed through the centre of a scraper ring in inner diameter of $2580.32 \mathrm{~mm}$, followed by drying under $120^{\circ} \mathrm{C}$ in a 2-metre-long air-dry oven. The moving speed of 259 yarns was $10 \mathrm{~m} / \mathrm{min}$. Coating process was conducted for three times to prepare luminescent warp 260 in diameter of $\sim 0.3 \mathrm{~mm}$. To achieve water resistance, silicone protective layer (Dow Corning, 12612577 ) could be further dip-coated on the luminescent warps.

\section{Fabrication of the display textile}

263 The weaving operation of the display textile was made on a rapier loom (Tong Yuan Textile 264 Machinery Co., Ltd.). The weave diagram was presented in Fig. 1a. Note that other fibre materials 
such as polyurethane-coated metal wire can be also co-woven inside.

\section{Structure and performance characterization of EL units}

267 The cross-sectional image of the single EL unit was obtained from scanning electron microscopy 268 (S-4800, Hitachi) operated at $1 \mathrm{kV}$. The transparency of the ionic gel was characterized by an UV269 visible spectrophotometer (UV-2550 SHIMADZU Spectrometer) to scan wavelengths from 450 to

$270700 \mathrm{~nm}$. The luminance of a single EL unit was detected by a spectrophotometer (Photoresearch 271 PR-680) under an alternating voltage supplied by a function waveform generator (Keysight 272 33500B Series) connected with a high-voltage power amplifier (610 E, TREK Inc.). If not 273 specified, the test parameters of the EL unit were $1.2 \mathrm{~V} / \mu \mathrm{m}$ and $2 \mathrm{kHz}$, and the intersection area in 274 EL unit projection was used as effective device area. The voltage, current and power consumption 275 were measured (Keysight 34461 A Digit multimeter) using a test circuit (see Extended Data Fig. $2763 \mathbf{d}$ for details). The EL mappings of EL units under bending, sliding and rotating were obtained by 277 mapping the photographs according to the gray value in Matlab. For statistical analysis of relative 278 EL intensity of the units in the display textile, the gray values of the units were extracted from the 279 photographs by ImageJ, which had been captured by a digital camera (D3400, NIKON) in dark 280 room. Uniformity of EL unit array was evaluated according to relative deviation (RD) calculated 281 by $R D=\left(L_{x}-\bar{L}\right) \bar{L} \times 100 \%$, where $\mathrm{L}_{\mathrm{x}}$ was the EL intensity of a single EL unit, and $\overline{\mathrm{L}}$ was the 282 average intensity of 600 units. Stability of EL unit array was evaluated by counting the EL intensity 283 variation (calculated as $\mathrm{L} / \mathrm{L}_{0}$, where $\mathrm{L}_{0}$ and $\mathrm{L}$ were the intensities before and after deforming, 284 respectively) of 600 EL units. The temperature changes of EL units were measured by an infrared 285 camera (PI 640, Optris).

\section{Washing test of display textile}

287 The washing tests were conducted in a standard washing machine (SW-12E, Nantong Hongda 288 Experimental Instrument Co., Ltd.) (Extended Data Fig. 6d). Each EL unit was woven into a $2 \times 2$ $289 \mathrm{~cm}$ textile and put into individual washing chamber containing $200 \mathrm{~g}$ water and $0.8 \mathrm{~g}$ detergent. 290 After washed for $30 \mathrm{~min}$ in room temperature, the textiles were rinsed under flowing water and 291 dried under $60{ }^{\circ} \mathrm{C}$ for $1 \mathrm{~h}$. The test parameters of EL performance were $3 \mathrm{~V} / \mu \mathrm{m}$ and $2 \mathrm{kHz}$. 


\section{Calculation of power consumption of an EL unit}

293 The voltage at the certain position (the root mean square at A, B, C, Ground, referred to Extended

294 Data Fig. 3d) and the resistance of each resistor were firstly measured. The current across each 295 resistor was calculated as $I=V / R$. The current through Resistors 2 and 3 was:

$$
I_{2}=\frac{V_{A B}}{R_{2}}=I_{3}
$$

297 Using Equation 1, $\mathrm{V}_{\mathrm{AC}}$ was calculated (the voltage across the entire test circuit):

$$
V_{A C}=V_{A B}+V_{B C}=I_{2} R_{2}+I_{3} R_{3}
$$

299 The current across $R_{1}$ was equal to the total current through the test circuit. Based on this equality, 300 the power of the test circuit was:

$$
P_{\text {total }}=I_{1} V_{A C} \cos \theta
$$

302 where $\theta$ represented the phase shift between the current and voltage waveforms across the test 303 circuit. This phase shift was measured using an oscilloscope (TDS 2012C, Tektronix). Hence, the 304 real power of the test circuit, which included energy used by the EL unit and the resistors, could 305 be calculated according to:

$$
P_{\text {total }}=P_{\text {unit }}+P_{\text {resistor }}
$$

307 The power consumption of the EL unit was obtained by subtracting the power consumption of the 308 resistor. The power consumed by each resistor was calculated by the equation of $P=I^{2} R$.

\section{Electric-field simulation of the EL unit through finite element method}

310 The EL unit was constructed in ABAQUS CAE with geometry characteristics in Extended Data

311 Fig. 1i. 8-node linear reduced-integration hybrid brick elements (C3D8RH) were used to model 312 the transparent conductive weft of hyperelastic materials. Through mesh convergence study, 30284 313 and 39840 elements were generated for the luminescent warp and the transparent conductive weft, 314 respectively.

316 Mechanical properties of materials were defined by directly importing the uniaxial tensile test data 317 (Extended Data Fig. 1). Linear elastic model was employed for luminescent warp. Ogden 
318 hyperelastic model was employed for the polyurethane ionic gel fibre with the strain energy 319 potential function $\mathrm{W}$ :

$$
W=\frac{2 \mu_{1}}{\alpha_{1}^{2}}\left(\bar{\lambda}_{1}^{\alpha_{1}}+\bar{\lambda}_{2}^{\alpha_{1}}+\bar{\lambda}_{3}^{\alpha_{1}}-3\right)+\frac{1}{D_{1}}(J-1)^{2}
$$

321 Where $\bar{\lambda}_{i}$ is the deviatoric principal stretches $\bar{\lambda}_{i}=J^{-\frac{1}{3}} \lambda_{i}$. Here $\lambda_{i}$ is the principal stretches. This

322 form could be degenerated into Neo-Hookean form of potential energy when $\alpha_{1}=2$.

324 As mentioned above, periodic boundary conditions were imposed along the axial direction of the 325 transparent conductive weft. The axial length of EL unit was fixed since the weft was kept tight 326 during weaving process. Contact between transparent conductive weft and luminescent warp was 327 defined as default hard contact. The loads imposed on both ends of the transparent conductive weft 328 were estimated by outputting the reaction force of the polyurethane ionic gel fibre under a 329 displacement of $0.48 \mathrm{~mm}$.

330 Static electric analyses were then conducted on the deformed models to obtain the electric fields 331 in the $\mathrm{ZnS}$ phosphor layer. The transparent conductive weft was grounded and $90 \mathrm{~V}$ electric 332 potential was imposed on the core conductive yarn of luminescent warp. The dielectric constant 333 of the luminescent layer was $3.621 \times 10^{-11} \mathrm{~F} / \mathrm{m}$.

\section{$334 \quad$ Fabrication of textile keyboard}

335 The textile keyboard was based on a jacquard method by weaving carbon fibres (1K, TORAY, 336 Japan), silver-plated yarns and cotton yarns according to the weave diagram in Extended Data 337 Fig. 7a.

339 Ti wire (diameter of $127 \mu \mathrm{m}$, Alfa Aesar) was used as the substrate of photoanode. First, the Ti 340 wire was sequentially cleaned by sonication in deionized water, acetone and isopropanol for $5 \mathrm{~min}$ 341 each. Then $\mathrm{TiO}_{2}$ nanotubes were grown on $\mathrm{Ti}$ wire by an anodic oxidation in a water bath. A 0.3 $342 \mathrm{wt} \% \mathrm{NH}_{4} \mathrm{~F} /$ ethylene glycol (Sinopharm) solution containing $8 \mathrm{wt} \% \mathrm{H}_{2} \mathrm{O}$ was prepared as the 343 electrolyte. The growth was operated in a two-electrode system with Ti wire as anode and Pt plate 344 as cathode at $60 \mathrm{~V}$ for $2 \mathrm{~h}$. The modified Ti wire was washed and annealed at $500{ }^{\circ} \mathrm{C}$ for $60 \mathrm{~min}$. 
345 After cooled to $110^{\circ} \mathrm{C}$ in the furnace, the wire was immersed in Z907 (Shanghai MaterWin New 346 Materials Co., Ltd.) solution (0.3 mM, mixture solvent of dehydrated acetonitrile (Adamas) and 347 tert-butanol (Sinopharm) with an equal volume ratio) for $16 \mathrm{~h}$. Next, CuI was drop-coated onto the 348 modified $\mathrm{Ti}$ wire in a glovebox at $110^{\circ} \mathrm{C}$. CuI solution was prepared by dissolving $0.16 \mathrm{M}$ cuprous 349 iodide (Aladdin), 1-methyl-3-ethylimidazolium thiocyanate (Lanzhou Greenchem ILs) and 0.2 $350 \mathrm{mM}$ 4-tert-butylpyridine (Adamas) in acetonitrile.

352 The aqueous zinc ion battery fibre was composed of a $\mathrm{MnO}_{2}$ coated carbon nanotube (CNT) fibre 353 cathode, a zinc wire anode, and gelation $/ \mathrm{ZnSO}_{4}$ water-based gel electrolyte ${ }^{29}$. A CNT fibre was 354 first synthesized by floating-catalyst method ${ }^{30}$. For the fibre cathode, $\mathrm{MnO}_{2}$ was electrodeposited 355 onto the CNT fibre through a scalable electrodeposition method (pulse mode $1.5 \mathrm{~V}$ for $1 \mathrm{~s}$ and 0.7 $356 \mathrm{~V}$ for $10 \mathrm{~s}$ ) in electrolyte containing $0.1 \mathrm{M} \mathrm{Mn}(\mathrm{Ac})_{2} \cdot 4 \mathrm{H}_{2} \mathrm{O}$ (Aladdin) and $0.1 \mathrm{M} \mathrm{Na}_{2} \mathrm{SO}_{4}$ 357 (Sinopharm) with an $\mathrm{Ag} / \mathrm{AgCl}$ reference electrode and a $\mathrm{Pt}$ counter electrode. The $\mathrm{MnO}_{2}$ loading 358 mass was $0.5 \mathrm{mg} / \mathrm{cm}$ for the cathode fibre. The zinc wire with a diameter of $\sim 0.5 \mathrm{~mm}$ was polished 359 and rinsed before use. The cathode and anode wires were uniformly coated with gel electrolyte 360 and then twisted together. The gel electrolyte was prepared by firstly dissolving $1.0 \mathrm{~g}$ gelatin 361 (Sinopharm) and $0.1 \mathrm{~g} \mathrm{Na}_{2} \mathrm{~B}_{4} \mathrm{O}_{7}$ (Aladdin) in $10 \mathrm{~mL}$ deionized water at $80{ }^{\circ} \mathrm{C}$. Then $10 \mathrm{mM}$ $362 \mathrm{ZnSO}_{4} \cdot 7 \mathrm{H}_{2} \mathrm{O}$ (Aladdin) and $1 \mathrm{mM} \mathrm{MnSO}_{4} \cdot \mathrm{H}_{2} \mathrm{O}$ (Aladdin) were added under stirring until a 363 homogenous solution was formed. The as-fabricated battery was dried at room temperature, and 364 the gel electrolyte concurrently served as electrolyte and separator. The battery was put into a 365 flexible poly (vinyl chloride) tube and sealed by resin adhesive at the terminals of the tube.

367 Silver-plated nylon yarns were woven in the warp direction as the counter electrodes for energy 368 harvesting part and the electrical connections for energy storing part. The cotton threads, modified 369 photoanode fibres and zinc-ion battery fibres were then alternately woven in the weft direction. 370 Current density-voltage curves of the energy harvesting part were recorded by a Keithley 2400 371 Source Meter under the illumination $\left(100 \mathrm{~mW} / \mathrm{cm}^{2}\right)$ of simulated AM1.5 solar light from a solar 372 simulator (Oriel-Sol3A 94023A equipped with a $450 \mathrm{~W}$ Xe lamp and an AM1.5 filter). 373 Electrochemical measurements were performed on an electrochemical workstation (CHI 660a).

\section{$374 \quad$ Fabrication of the integrated textile system}


375 Different electronic textiles were arranged on a piece of cloth by changing functional fibres during 376 weaving process, which were integrated on a jacket by hot melt adhesive or sewing. The 377 microcontroller of textile electronics was STM32F103T8U6, an ARM32-bit CortexTM-M3 CPU 378 with QFN36 package (DM14580). By using an analog-to-digital converter to sample the keyboard 379 resistance, the single bus detection of the keyboard was realised. Driving circuit of display textile 380 was provided by Shanghai Mi Fang Electronics Co., Ltd. The communication between integrated 381 textile system and mobile phone was realised by Bluetooth module (HC-05). If necessary, the other 382 commercial portable power source could be also integrated as backup power.

\section{Collection and decoding of electroencephalogram signals}

384 The signals of volunteers were collected by the wearable recorder (MindWave Mobile 2, 385 Neurosky). The volunteers were asked to play a car race game to be in an anxious mental state and 386 lay back in meditation to be in a relaxed mental state. The signals were recorded in real time and 387 collected on a computer. After downsampled to $100 \mathrm{~Hz}$, the signals were filtered by 4th-order IIR 388 bandpass filters with bandwidth of $0.1-48 \mathrm{~Hz}$. The time domain signals were transferred to 389 spectrogram by fast Fourier transform.

\section{Data availability}

391 All data are contained within the manuscript. Raw data are available from the corresponding 392 authors upon reasonable request.

\section{Code availability}

394 Custom code used in this study is available from the corresponding authors upon reasonable 395 request.

397 29. Wang, Z. F. et al. A flexible rechargeable zinc-ion wire-shaped battery with shape memory 398 function. J. Mater. Chem. A 6, 8549-8557 (2018).

399 30. Lee, J. et al. Direct spinning and densification method for high-performance carbon nanotube 400 fibers. Nat. Commun. 10, 2962 (2019). 


\section{Acknowledgements}

403 This work was supported by MOST (2016YFA0203302), NSFC (21634003, 51673043, 21805044, 404 21875042), STCSM (18QA1400800, 19QA1400800), SHMEC (2017-01-07-00-07-E00062), and 405 Yanchang Petroleum Group. Part of the sample fabrication was performed at Fudan Nano406 fabrication Laboratory. We thank Shanghai Mi Fang Electronics Co., Ltd. for technical support of 407 the display driving circuits, Idea Optics Co., Ltd. for offering test instruments, Jiaying Zhao for 408 assistance in textile weaving, and Ai Lin Chun of Science Storylab for critically reading and editing 409 the manuscript.

411 Author contributions

412 H. P. and P. C. conceived and designed the research project. X. Shi. and Y. Z. and P. Z. performed 413 the experiments on the display textile, keyboard and integration systems and contributed equally 414 to this work. J. S. and Y. Y. and Q. T. performed the simulation. Z. G. performed the experiments 415 on photovoltaic textiles. M. L. and J. W. performed the experiments on energy storage fibres. X. 416 Shi. and Y. Z. and P. Z. and X. X. analysed the data. B. Z., X. Sun., L. Z., Q. P., D. J. and all other 417 authors discussed the data and wrote the paper.

\section{Competing interests}

420 The authors declare no competing interests.

422 Additional information

424 Supplementary Information is available for this paper.

426 Correspondence and requests for materials should be addressed to $\mathrm{P}$. C. 427 (peiningc@fudan.edu.cn) and H. P. (penghs@fudan.edu.cn). 
a
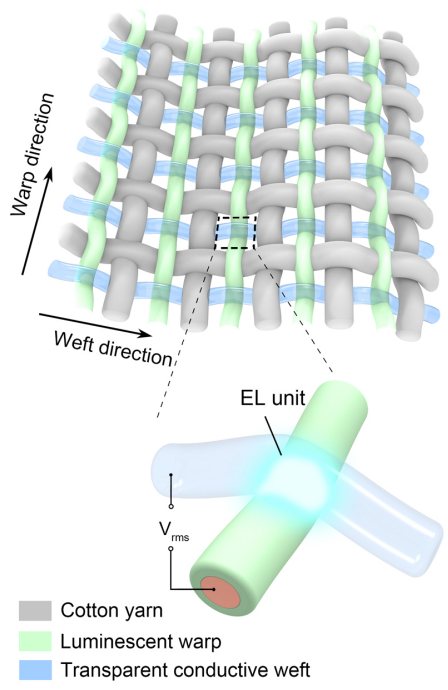

b
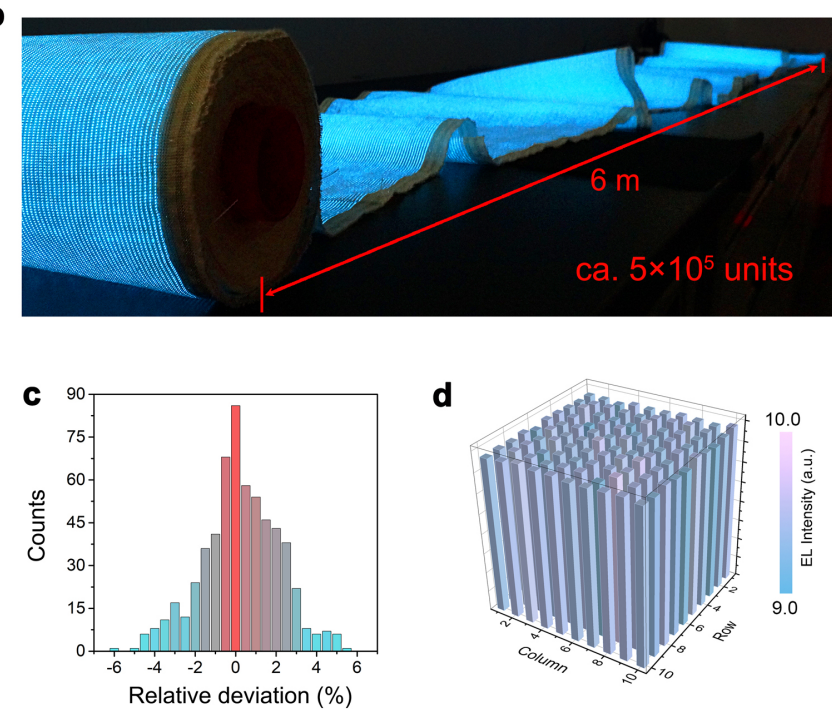

d

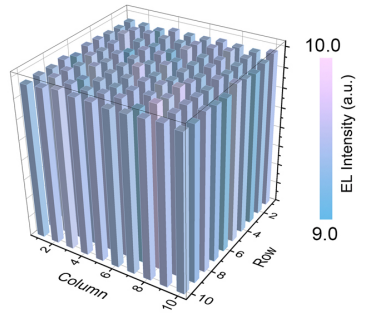

e

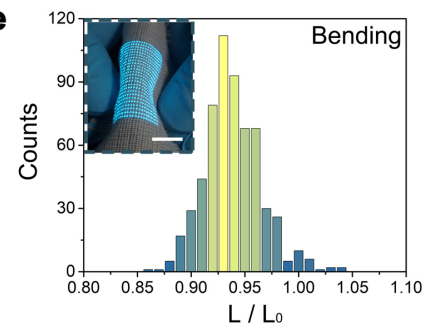

h

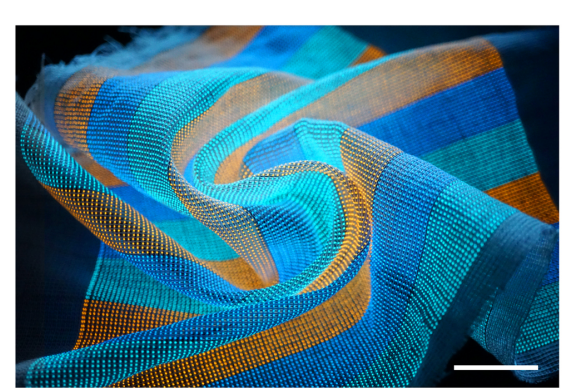

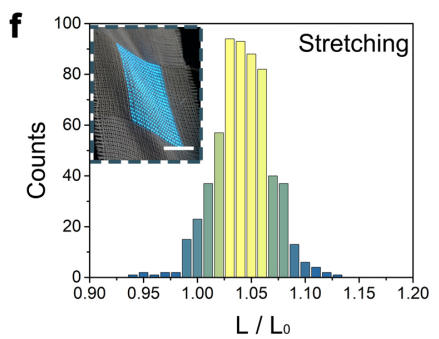

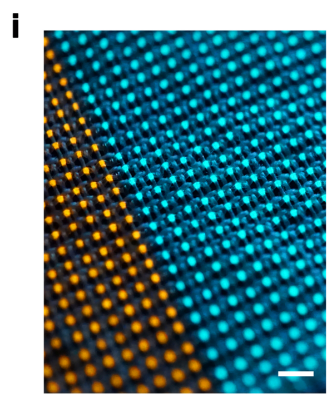

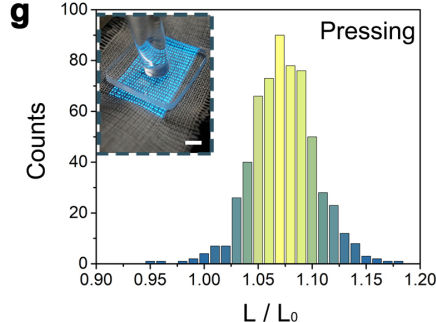

L / Lo

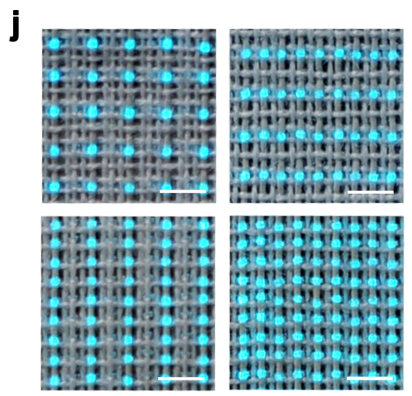

434 Fig. 1 | Structure and EL performance of the display textile. a, Schematic showing the weave

435 diagram of the display textile. Each contacting luminescent warp and transparent conductive weft

436 forms an EL unit (inset). Applied alternating voltage $\left(\mathrm{V}_{\mathrm{rms}}\right)$ turns on the EL units. b, Photograph

437 of a 6-metre-long display textile consisting of approximately $5 \times 10^{5}$ EL units. c, Statistical

438 distribution shows the relative deviations of emission intensity for $600 \mathrm{EL}$ units examined are

439 between $-6.3 \%$ and $5.2 \%$. Relative deviation is defined as deviation of luminance for a single EL

440 unit from the average value. d, Emission intensities of a $10 \times 10$ EL unit array is uniform $(<10 \%$ 
441 differences in intensity among the units). e-g, Statistical distribution shows minor $(<10 \%)$ 442 variations in luminance for $600 \mathrm{EL}$ units after 1,000 cycles of bending (e), stretching (f), and 443 pressing (g). Insets: photographs of tested samples. Scale bars, $1 \mathrm{~cm}$. h, Photograph of a functional 444 multicolour display textile under complex deformations, including bending and twisting. Blue and 445 orange are achieved by doping $\mathrm{ZnS}$ with copper and manganese, respectively. Scale bar, $2 \mathrm{~cm}$. i, 446 Magnified photograph of the multicolour display textile from $\mathbf{h}$ show EL units are uniformly 447 spaced at a distance of $\sim 800 \mu \mathrm{m}$. Scale bar, $2 \mathrm{~mm}$. j, Photographs of EL units spaced at different 448 distances obtained by changing the weaving parameters. Scale bars, $2 \mathrm{~mm}$. 

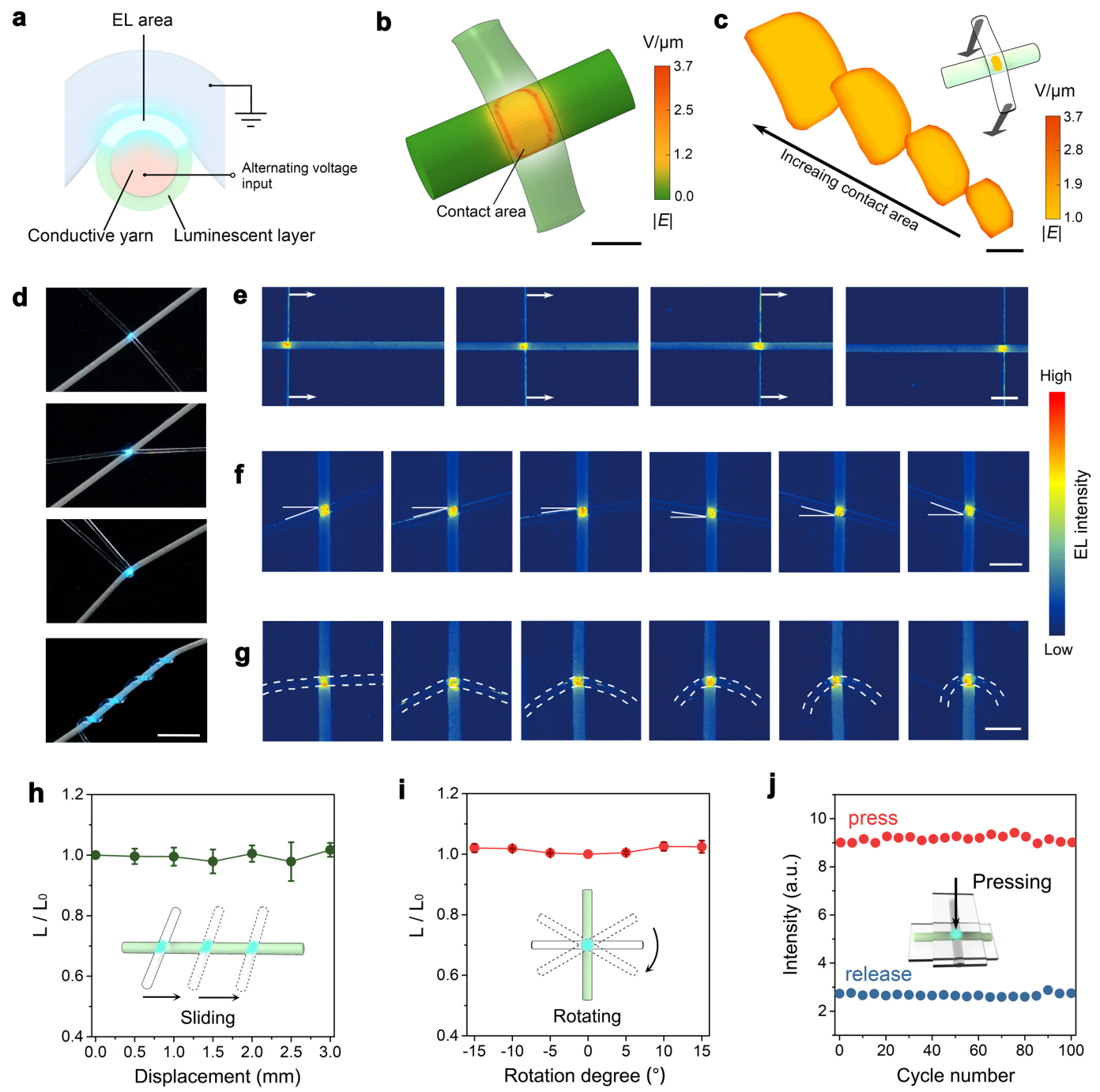

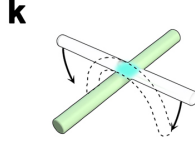

Weft bending
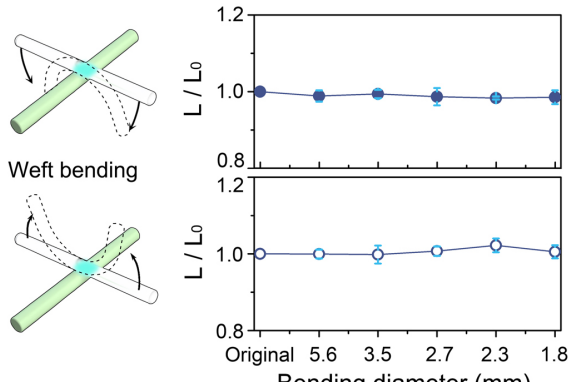

Bending diameter $(\mathrm{mm})$
I

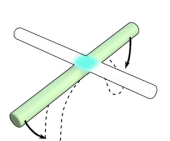

Warp bending
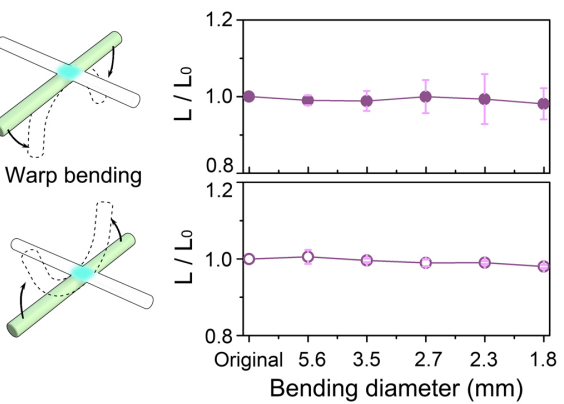

452 Fig. 2 Characterization of EL units of the display textile. a, Schematic of an EL unit formed

453 at the contact area between luminescent warp and transparent conductive weft. Light emission 454 occurs when an alternating electric voltage is applied. b, c, Simulation using finite element method 455 shows electric field distribution at the contact area in an EL unit is uniform (b) and does not change 
456 with increasing contact areas (c). d, Photographs show stable light emission as the transparent 457 conductive weft contacted, leaned, twisted and knotted with the luminescent warp (top to bottom). 458 Scale bar, $2 \mathrm{~mm}$. e-g, EL maps show brightness of EL units remained stable even when the 459 transparent weft is slid (e), rotated (f) and bent ( $(\mathrm{g})$ along the luminescent warp. Colour bar indicates 460 relative EL intensity. Scale bars, $1 \mathrm{~mm}$. h-l, Luminance varied minimally when the transparent 461 weft is moved by $3 \mathrm{~mm}$ along the luminescent warp (h) and rotated by different degrees $\left(\mathbf{i} ; 0^{\circ}\right.$ is 462 when the weft is perpendicular to the warp), and when the EL unit is pressed and released for 100 463 cycles (j), bent along the weft length ( $\mathbf{k})$ and along the warp length (l) with increasing bending 464 angles. $\mathrm{L}_{0}$ and $\mathrm{L}$ correspond to the EL intensity before and after deformation, respectively. Error 465 bars are standard deviations of the results from at least three samples. 
a

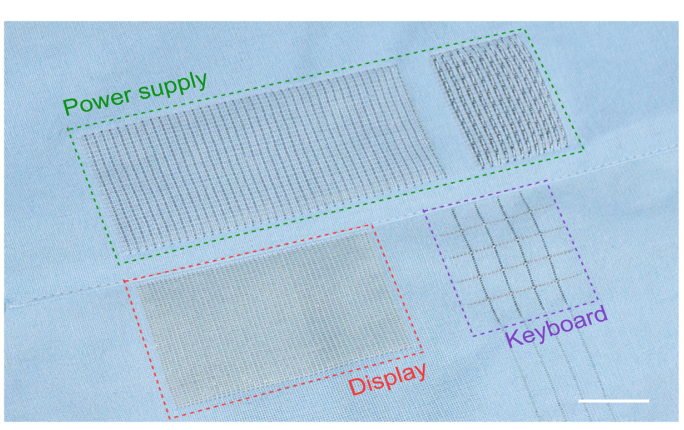

b

c

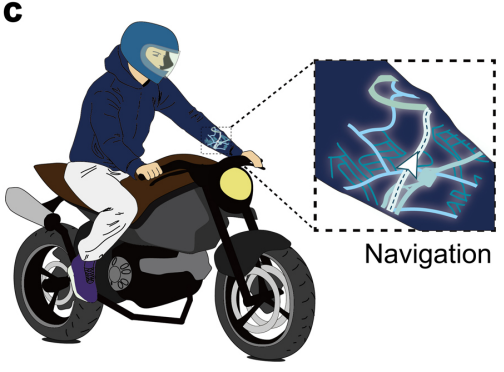

f

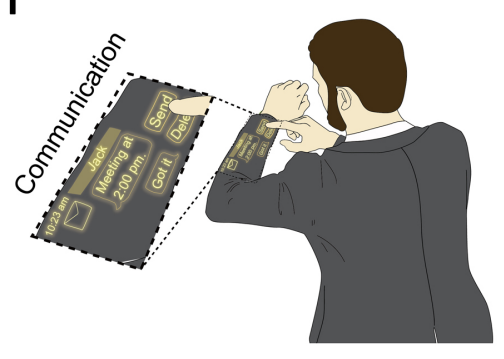

d
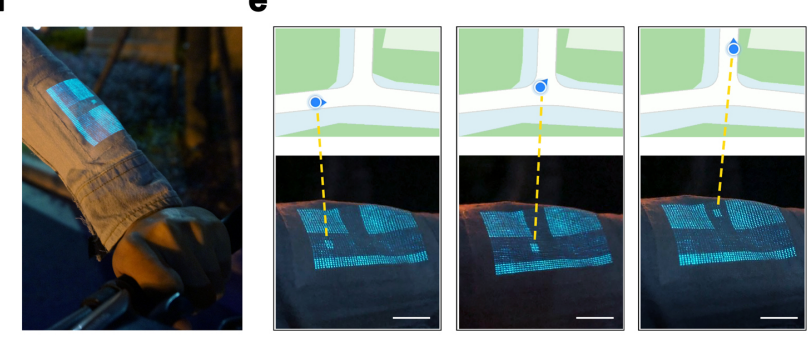

$\mathbf{g}$

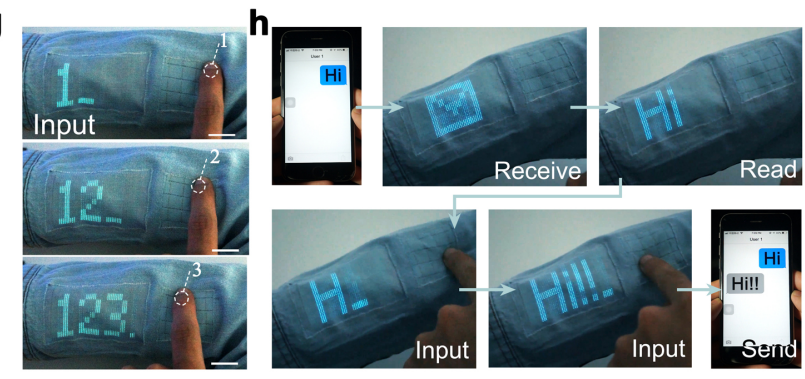

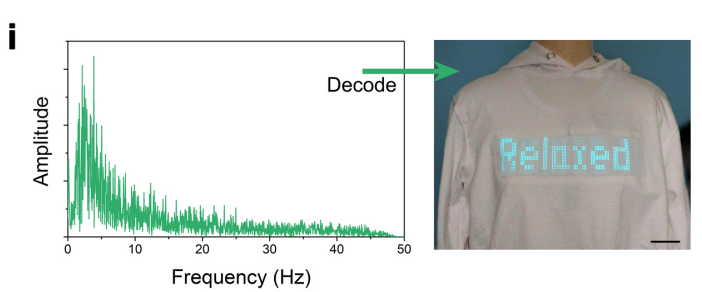

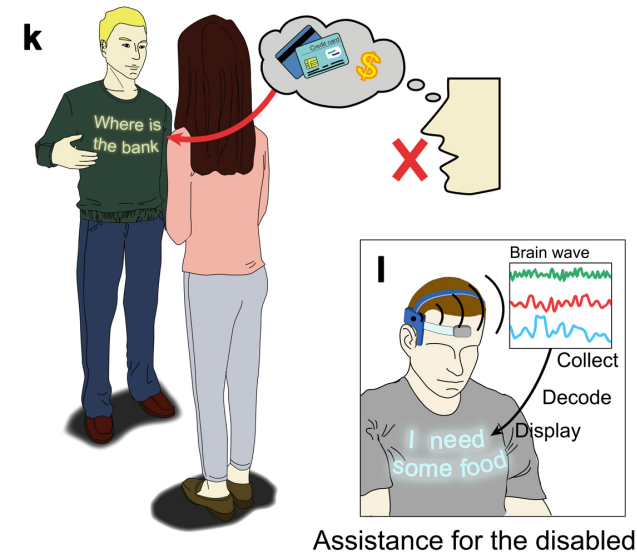

469 Fig. 3 | Application scenarios of integrated textile systems. a, Photograph of an integrated textile

470 system consisting of display, information input (keyboard) and power supply modules. Scale bar, $4712 \mathrm{~cm}$. b. System-level block diagram of the integrated textile system in a shows the modules 
472 connected to a microcontroller that is powered by solar energy harvesting and electrical energy 473 storing modules. c, Schematic illustrating the integrated textile as a smart node for the Internet of 474 Things to offer location services during driving. Selective illumination on the display module is 475 achieved by scanning electrical signals from the driver circuit row by row onto the array of EL 476 units. d, e, Real-time location at a T-junction on a sleeve, transferred through the Bluetooth and 477 microcontroller modules, is synchronized with the location map on a smartphone. Scale bars, 1 $478 \mathrm{~cm}$. f, Schematic shows textiles integrated with a display and keyboard can be used as a 479 communication platform. $\mathbf{g}$, Information is input onto clothes by pressing the keypress that is 480 woven into the textile. Scale bars, $2 \mathrm{~cm}$. h, Receiving and sending messages between the integrated 481 textile system and a smartphone. $\mathbf{i}, \mathbf{j}$, Expressions of mental states by decoding representative 482 electroencephalogram signals. The words "Relaxed" (i) and "Anxious" (j) are displayed on clothes 483 when the dominant brain waves are detected in the low-frequency region $(0-10 \mathrm{~Hz})$ and high484 frequency region (10-40 Hz), respectively. Scale bars, $5 \mathrm{~cm}$. $\mathbf{k}$, Display textiles could in the future 485 enable individuals with speech disorders to communicate and express themselves. I, Conceptual 486 image showing brain waves from a disabled person are being decoded into messages that are 487 displayed on a shirt made from an integrated textile. 
a

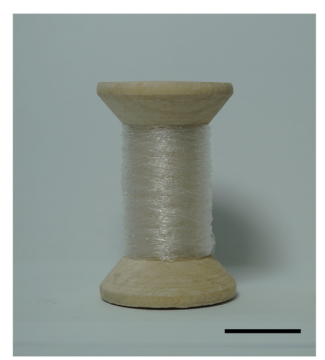

d
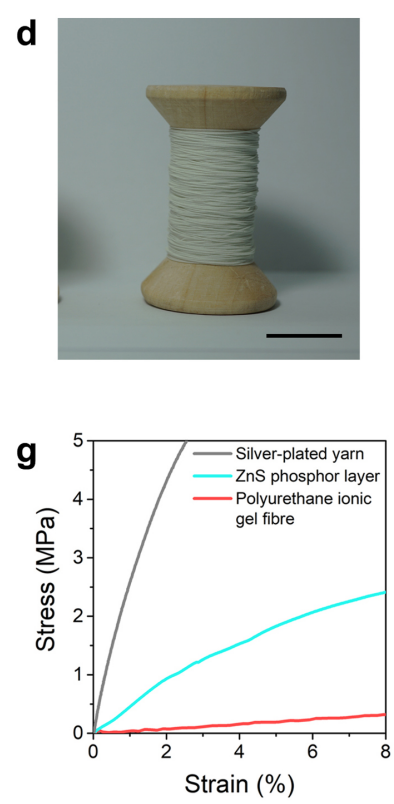

b
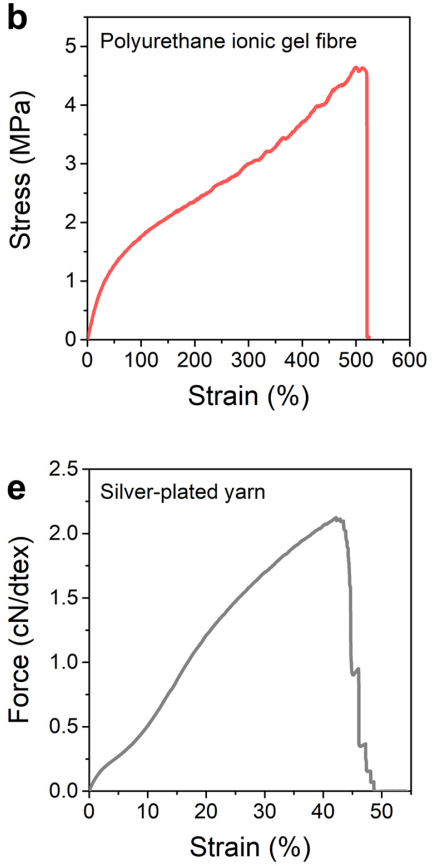

h

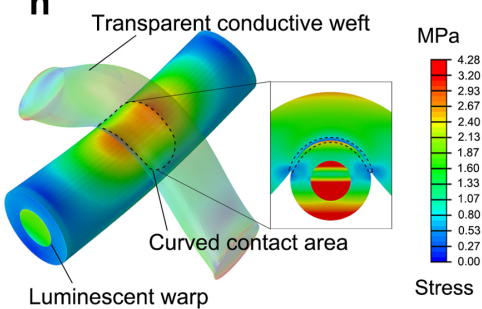

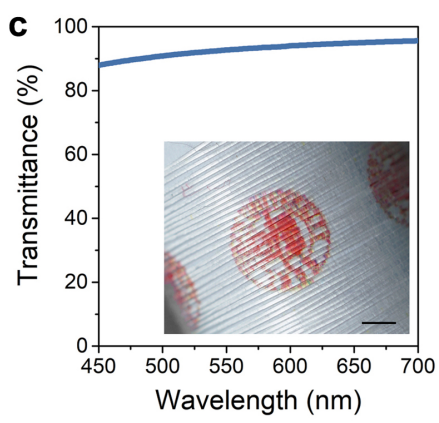
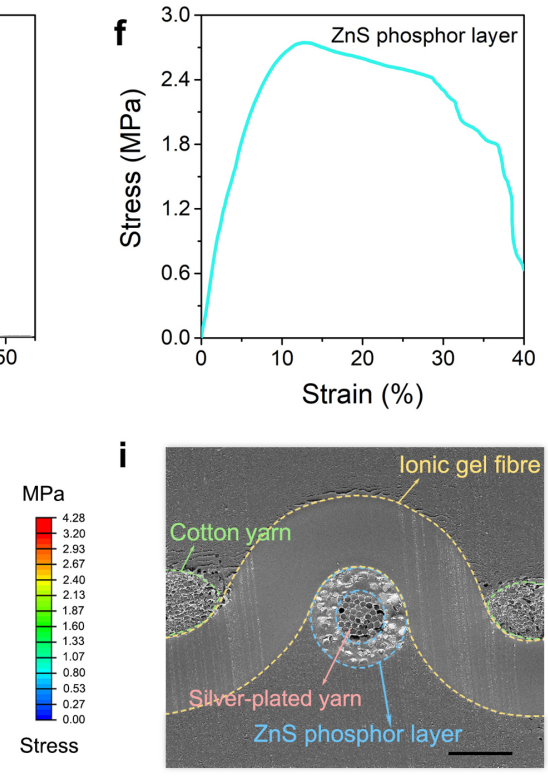

492 Extended Data Fig. 1 | Mechanical characterization of transparent conductive weft,

493 luminescent warp and their contact area. a, Photograph of transparent conductive wefts on a

494 spool. Scale bar, $2 \mathrm{~cm}$. b, Stress-strain curve of polyurethane ionic gel fibre. c, Transmittance of

495 ionic gel film with thickness of $250 \mu \mathrm{m}$. The inset is transparent conductive weft wound on a spool.

496 Scale bar, $2 \mathrm{~mm}$. d, Photograph of luminescent warps on a spool. Scale bar, $2 \mathrm{~cm}$. e, Force-strain

497 curve of silver-plated yarn. f, Stress-strain curve of $\mathrm{ZnS}$ phosphor layer. g, Comparison of 498 mechanical properties of silver-plated yarn, $\mathrm{ZnS}$ phosphor layer and polyurethane ionic gel fibre.

$499 \mathbf{h}$, Deformation and stress simulation in an EL unit. i, Cross-sectional SEM image of an EL unit 500 after embedded in resin. Scale bar, $200 \mu \mathrm{m}$. 
a

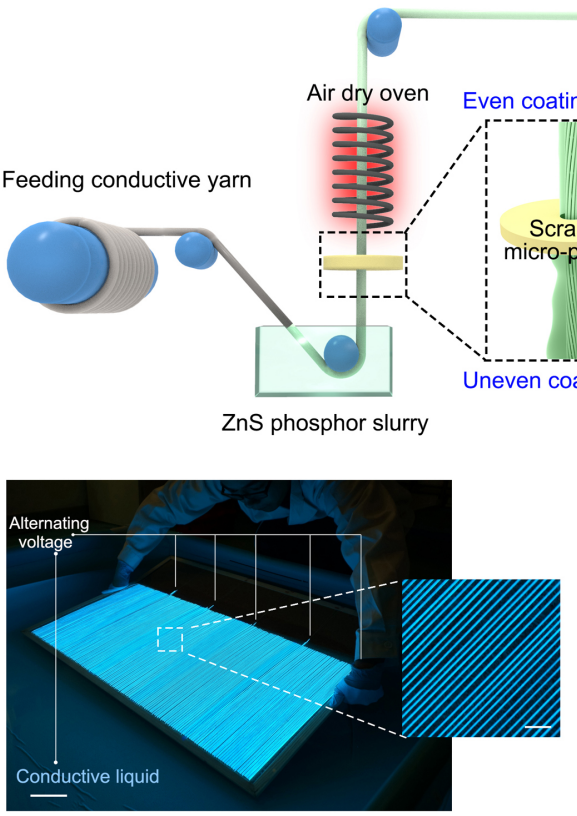

g

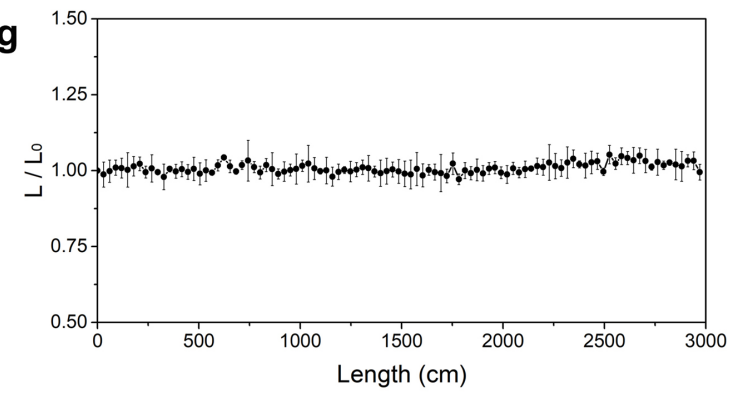

i

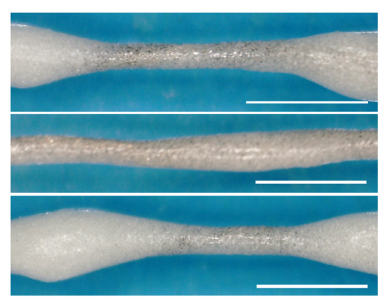

\section{j}

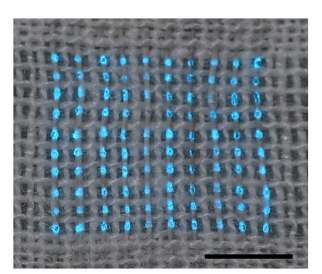

e

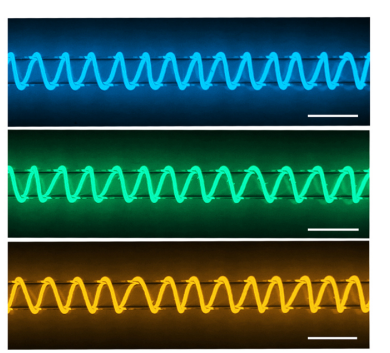

b

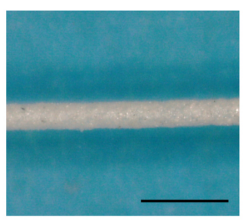

Winding luminescent warp

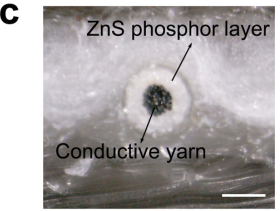

f

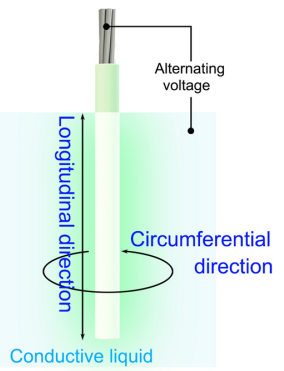

Conductive liquid
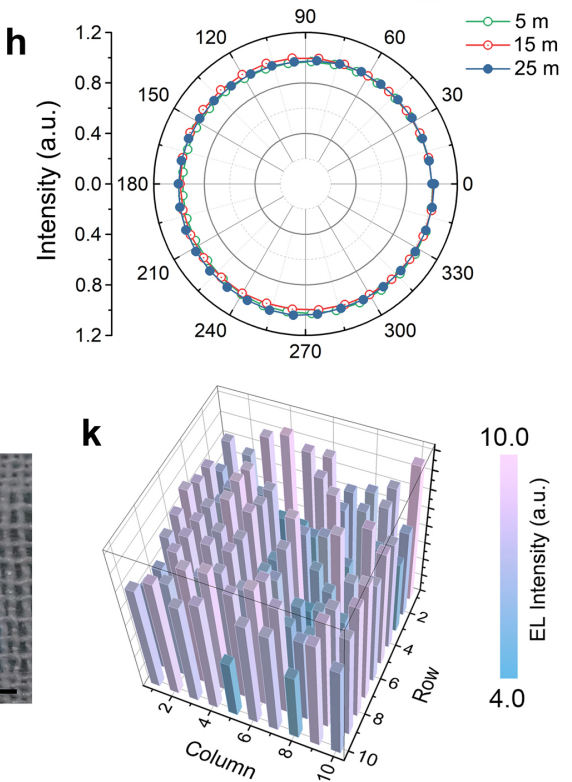

503 Extended Data Fig. 2 Longitudinally and circumferentially homogeneity of luminescent

504 warp. a, Schematic illustration of continuous fabrication of luminescent warp. b, Optical image 505 of luminescent warp. Scale bar, $1 \mathrm{~mm}$. c, Cross-sectional image of luminescent warp. Scale bar, $506200 \mu \mathrm{m}$. d, Photographs for $\sim 100$-metre-long luminescent warp parallelly arranged on a board in 507 a salt water pool. Scale bar, $10 \mathrm{~cm}$. The luminescent warp is lightened by applying alternating 508 voltage upon luminescent warp and salt water. The magnified area indicates the homogeneous 
509 luminescence along the fibre. Scale bar, $5 \mathrm{~mm}$. e, Multicolour luminescent warps wound on a glass 510 stick and lightened in salt water. Scale bars, $5 \mathrm{~mm}$. f, Schematic of longitudinal and circumferential 511 direction of luminescent warp. $\mathbf{g}$, Luminance distribution along the length of luminescent warp. 512 Error bars represent the standard deviations of the results from three samples. $\mathbf{h}$, Luminance 513 distribution around the luminescent warp circumference. i, Uneven luminescent layer in the case 514 that without using scraping micro-pinhole. Scale bars, $1 \mathrm{~mm}$. j, Photograph of the display textile 515 woven from luminescent warps with uneven coating. Scale bar, $5 \mathrm{~mm}$. k, Relative emission 516 intensities of a $10 \times 10$ EL unit array in $\mathbf{j}$.

517 

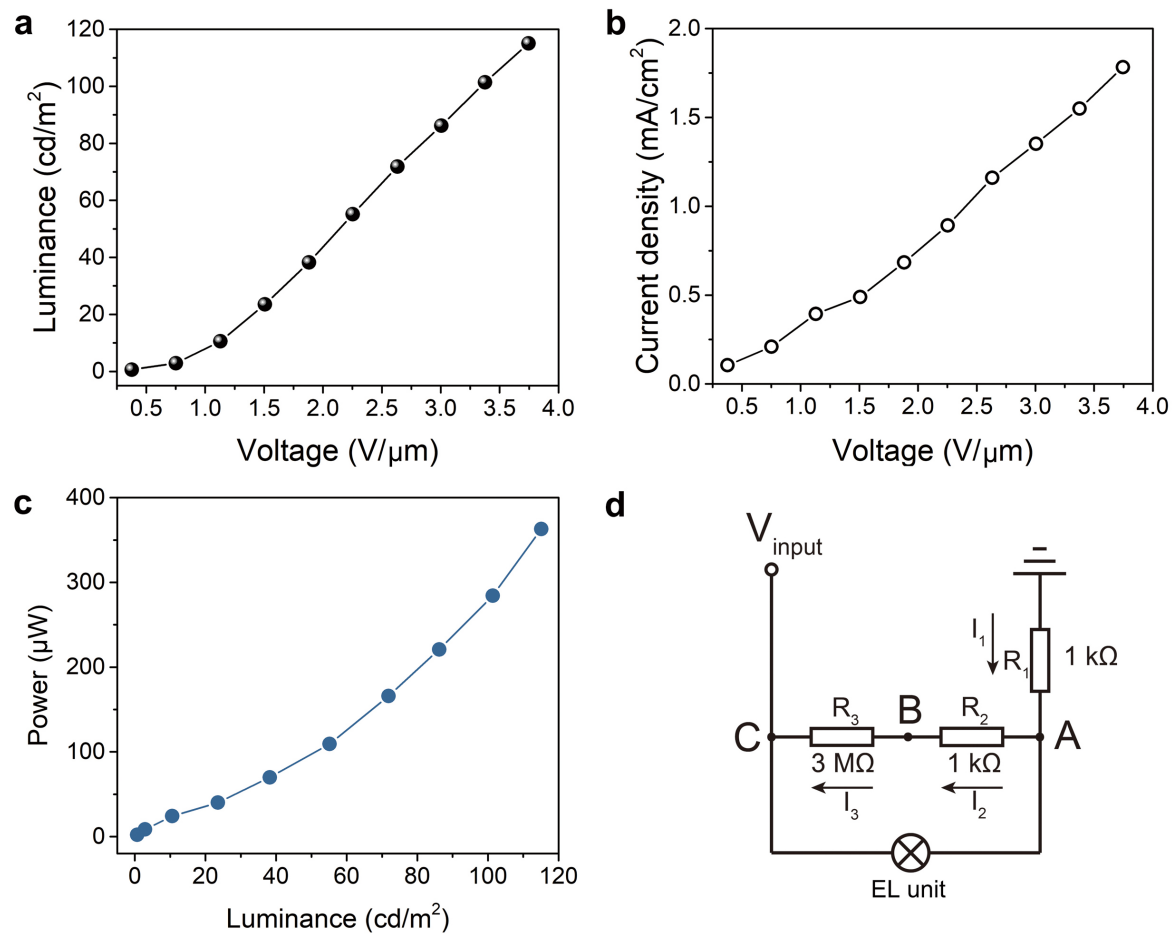

d
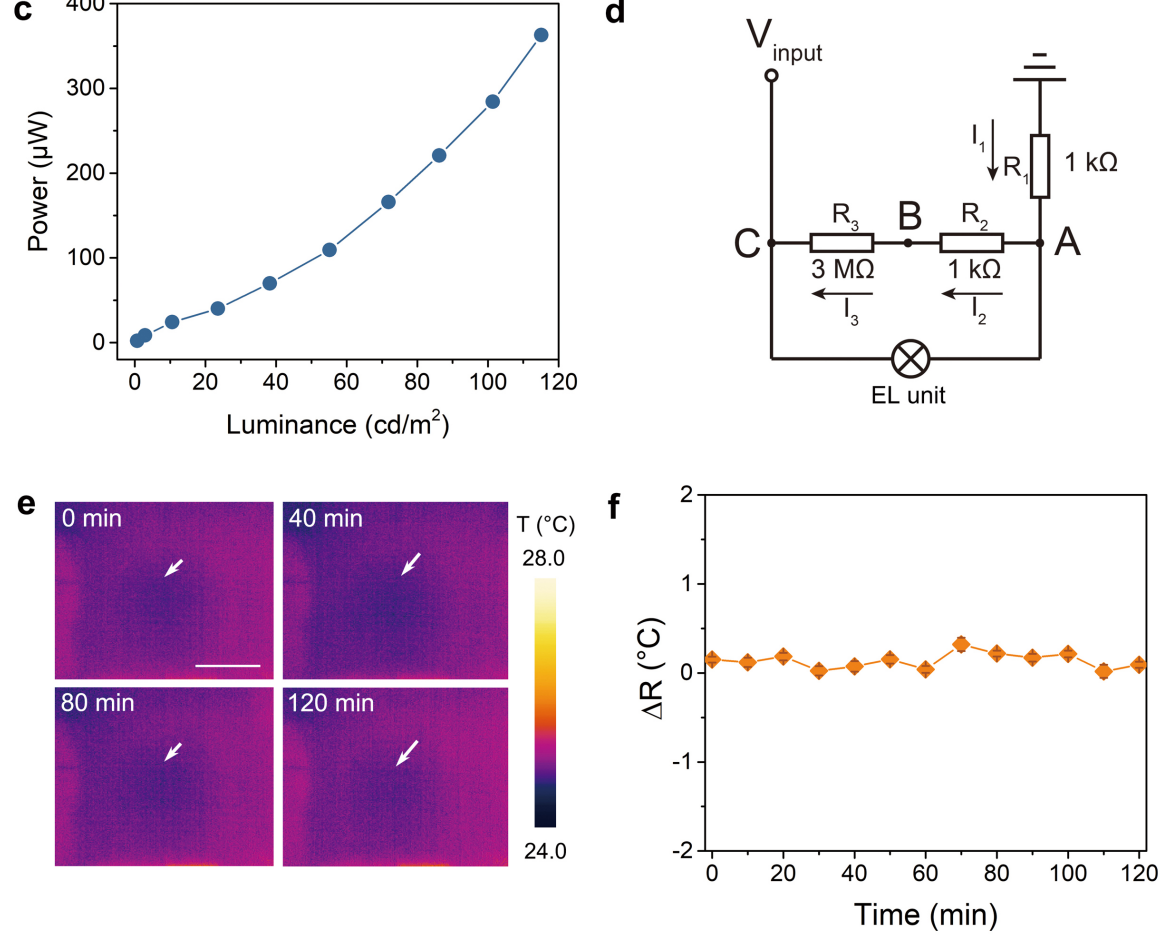

520 Extended Data Fig. 3 | EL performance of the EL unit. a-c, Luminance-voltage (a), current 521 density-voltage (b), and power-luminance (c) characteristics of the EL unit. d, Test circuit for 522 measuring power consumption of the EL unit. e, Thermal images of an illuminating EL unit along 523 increasing durations (under a power of $\sim 300 \mu \mathrm{W}$ ). The arrows indicate the position of the EL unit. 524 Scale bar, $5 \mathrm{~mm}$. f, Local temperature variations of EL units under a power of $\sim 300 \mu \mathrm{W}$. Error bars 525 represent the standard deviations of the results from five samples. 
a
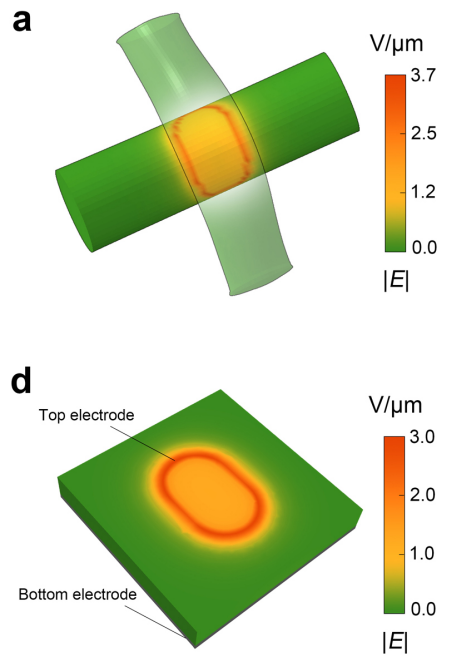

g

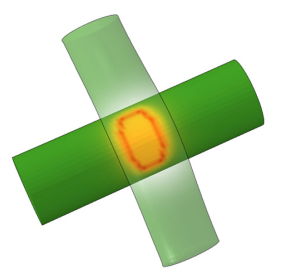

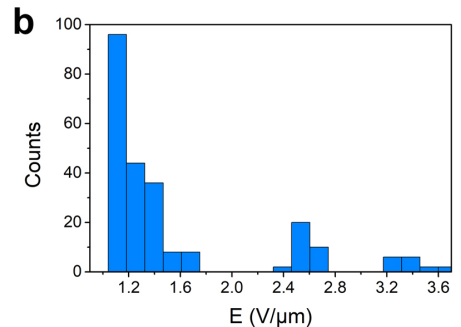

C
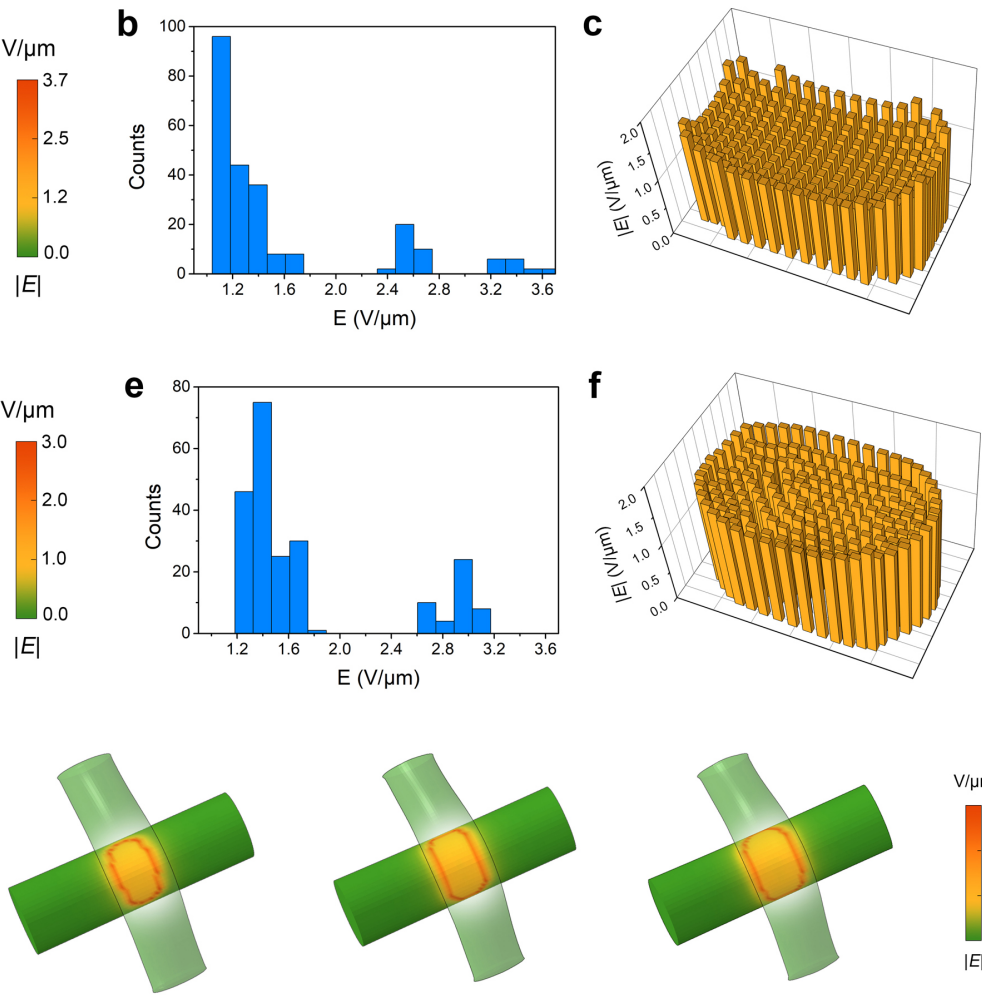

$\mathrm{V} / \mu \mathrm{m}$
$\prod_{0.0}^{3.7}$
2.5
1.2
$|E|$

528 Extended Data Fig. 4 | Comparison of electric field distribution of curved and planar contact

529 areas. Electric field distribution in woven EL unit (a-c) and traditional planar sandwiched EL 530 devices (d-f). a, d, Electric field distribution, b, e, statistics of the simulation elements on contact 531 area according to the electric field values, and $\mathbf{c}, \mathbf{f}$, visualization of electric field values by the 532 height of bars. g, Electric field distributions of EL unit along with increasing contact areas. 


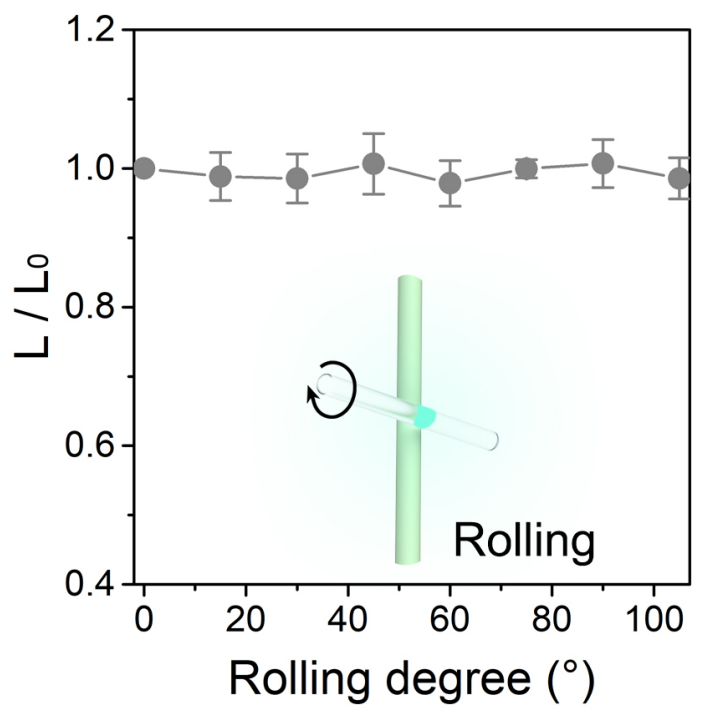

536 Extended Data Fig. 5 Luminance variations when the transparent conductive weft is rolled 537 around its central axis. Error bars represent the standard deviations of the results from three 538 samples. $\mathrm{L}_{0}$ and $\mathrm{L}$ correspond to the EL intensity before and after deformation, respectively. 

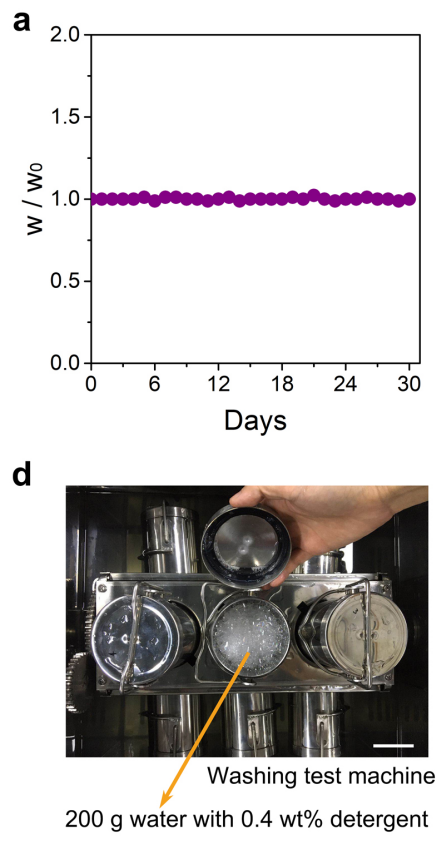

b

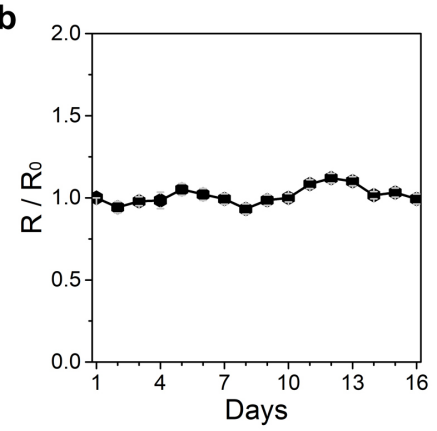

e

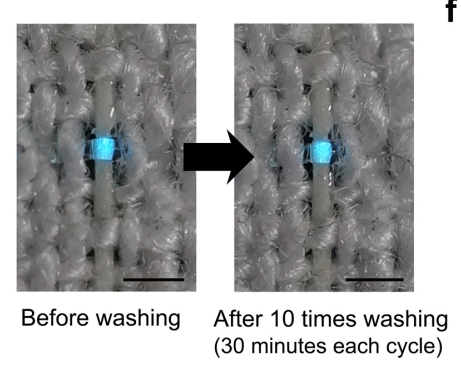

C

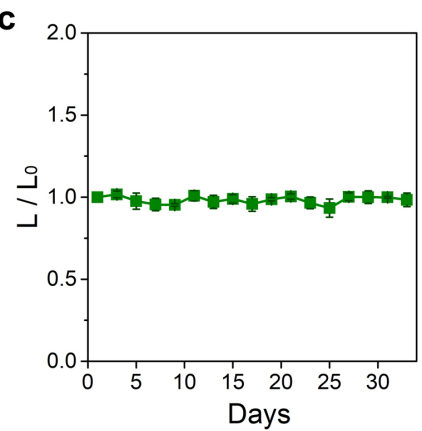

f

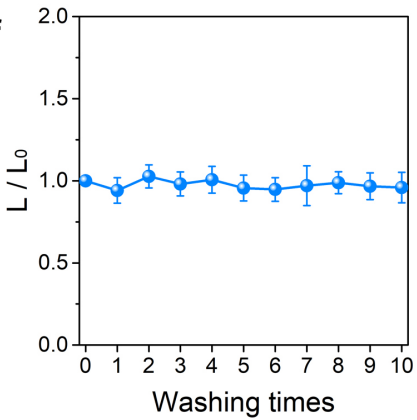

542 Extended Data Fig. 6 | Durability of polyurethane ionic gel fibre and EL units. a, b, Variation

543 of weight (a) and electrical resistance (b) for the polyurethane ionic gel fibre in open air at room

544 temperature. Here $\mathrm{w}_{0}$ and $\mathrm{w}$ correspond to the weights before and after exposure to the air,

545 respectively; $\mathrm{R}_{0}$ and $\mathrm{R}$ correspond to the electrical resistances before and after exposure to the air,

546 respectively. c, EL performance of EL units stored in open air. $\mathrm{L}_{0}$ and L correspond to the EL

547 intensity before and after exposure to the air, respectively. d, Photograph of inner structure of

548 washing machine. Scale bar, $5 \mathrm{~cm}$ e, Luminescence of EL unit before and after 10 cycles of 549 machine wash (30 minutes each cycle). Scale bar, $500 \mu \mathrm{m}$. f, Luminance variations when EL units 550 were washed and dried for 10 cycles. $\mathrm{L}_{0}$ and $\mathrm{L}$ correspond to the EL intensity before and after 551 washing, respectively. Error bars represent the standard deviations of the results from at least three 552 samples. 

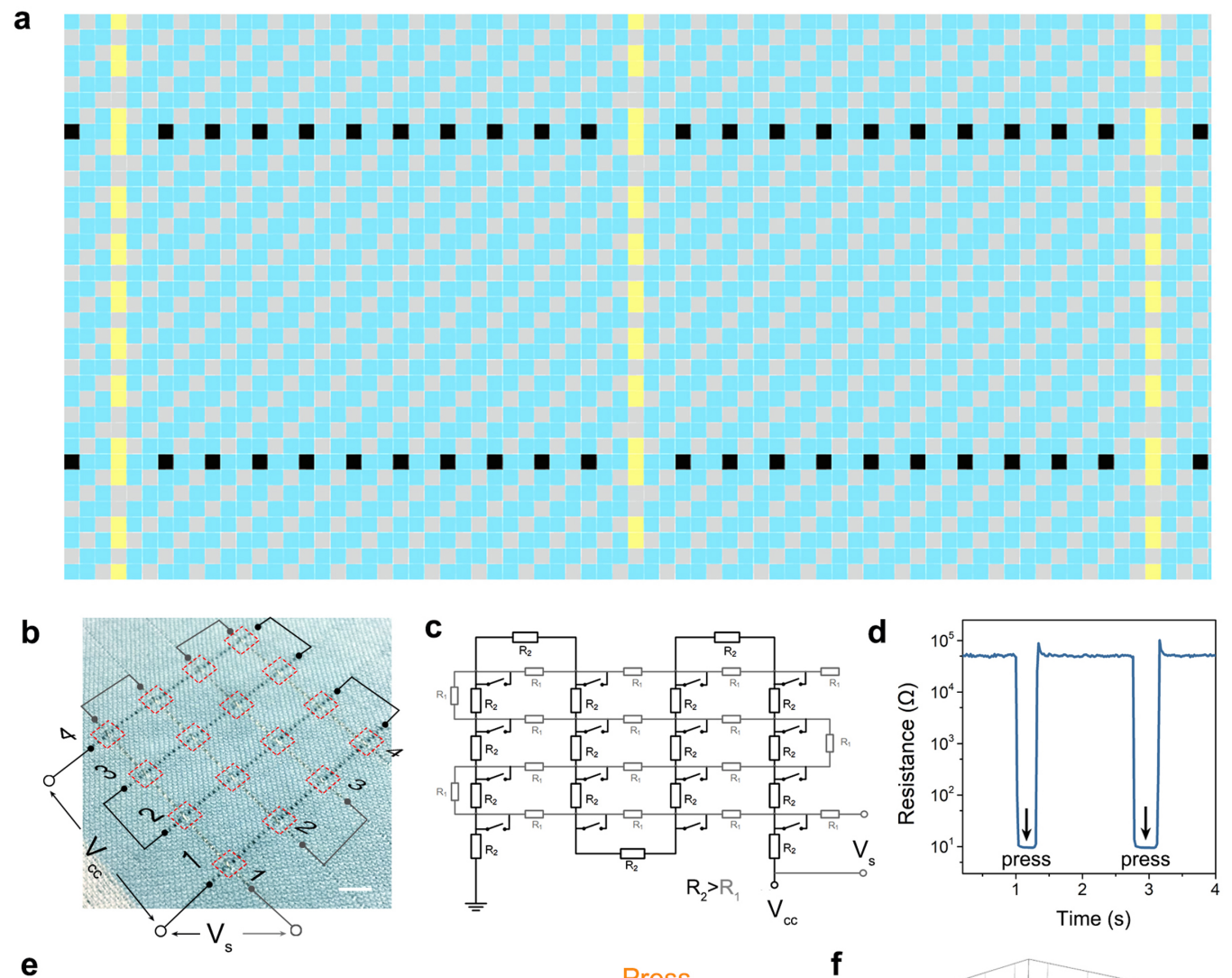

e
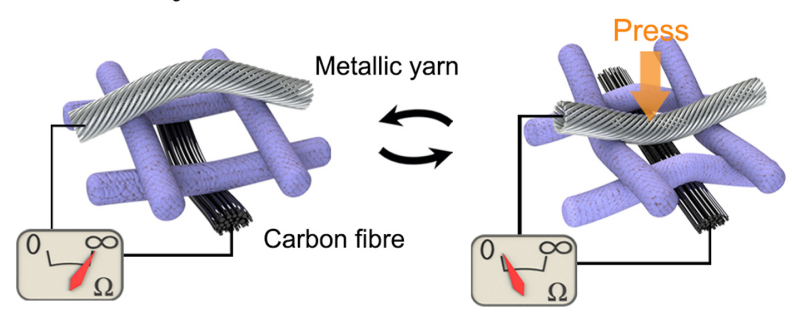

f

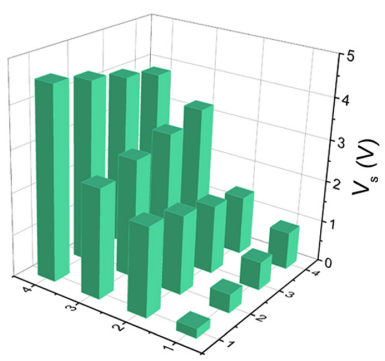

556 Extended Data Fig. 7 Characterization of textile keyboard. a, Weave diagram of the textile

557 keyboard (yellow: Ag-plated fibre, black: carbon fibre, blue: cotton yarn, gray: cotton yarn). b,

558 Photograph and electrical connection of a $4 \times 4$ textile keyboard. The red squares indicate the 559 positions of keypresses. Scale bar, $5 \mathrm{~mm}$. c, Equivalent circuit of a $4 \times 4$ keyboard. This keyboard 560 worked by reading the voltage between the metallic and carbon fibres (sample voltage, $\mathrm{V}_{\mathrm{s}}$ ) at an 561 applied voltage $\left(\mathrm{V}_{\mathrm{cc}}\right)$ of $5 \mathrm{~V}$. d, Pressing responses of a keypress with resistance variations that 562 were greater than 4 orders of magnitude. e, Working mechanism of the textile keyboard. f, Voltages $563\left(\mathrm{~V}_{\mathrm{s}}\right)$ recorded by pressing individual keypresses one by one. The correspondence between the 564 keypress position and its characteristic $\mathrm{V}_{\mathrm{s}}$ are indicated by the coordinates in $\mathbf{b}$ and $\mathbf{f}$. 
a
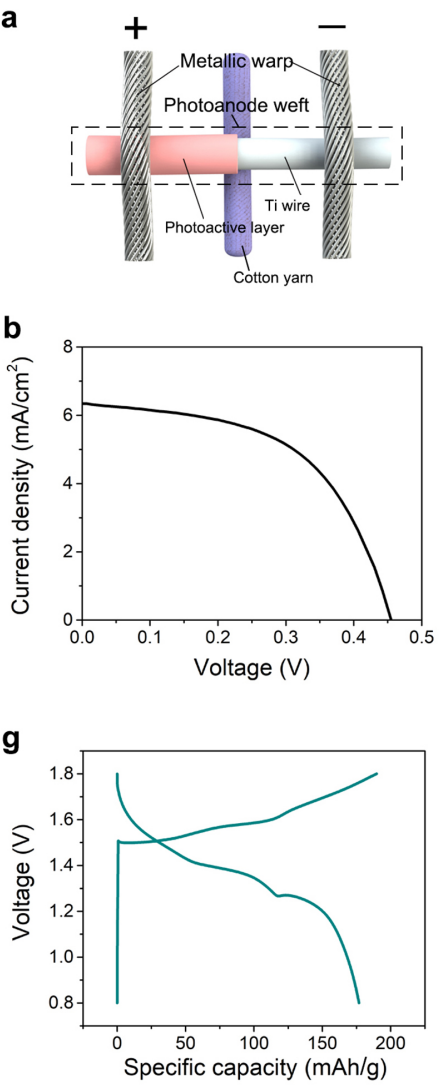

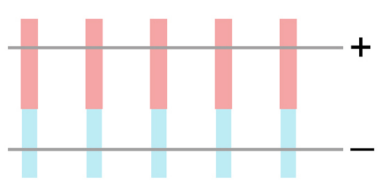

d

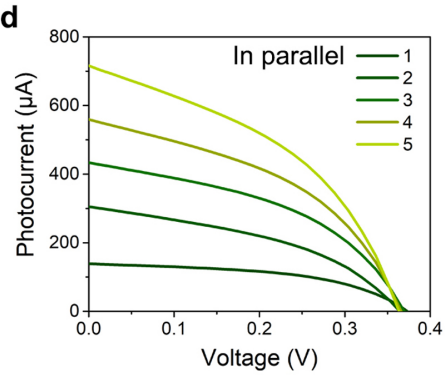

h

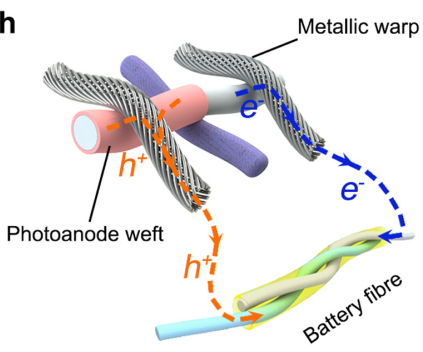

e
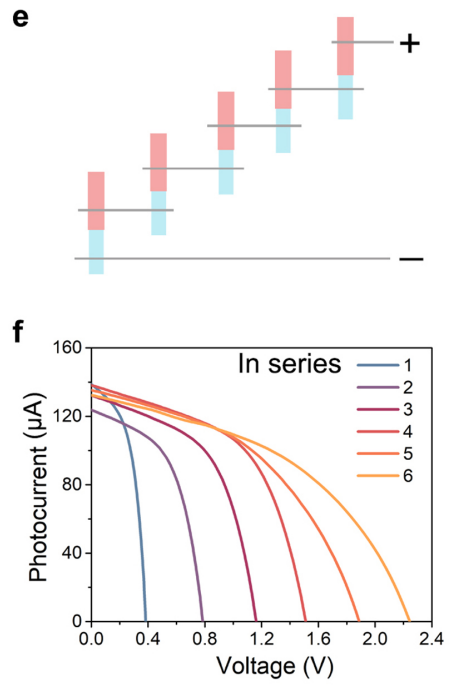

i

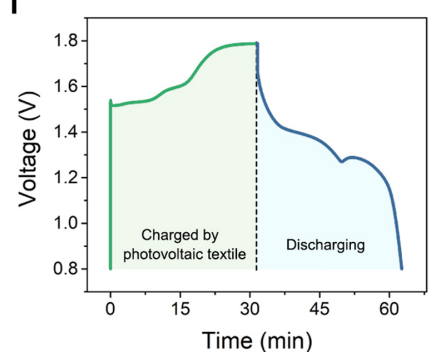

567 Extended Data Fig. 8 Characterization of textile power supply system. a, Schematic of a

568 woven photovoltaic unit. b, Current density-voltage characteristics of the photovoltaic unit, 569 exhibiting a short-circuit current density of $6.32 \mathrm{~mA} / \mathrm{cm}^{2}$ and an open-circuit voltage of $0.45 \mathrm{~V}$. c, 570 Schematic of the woven photovoltaic units connected in parallel. d, Current-voltage curve of the 571 photovoltaic textile with increasing numbers of photoanode wefts connected in parallel. e, 572 Schematic of the woven photovoltaic units connected in series. f, Current-voltage curve of the 573 photovoltaic textile with increasing numbers of photoanode wefts connected in series. g, 574 Galvanostatic charge/discharge curves at $200 \mathrm{~mA} / \mathrm{g}$ (based on the active material of cathode). The 575 battery fibre exhibited a mass capacity of $176.9 \mathrm{mAh} / \mathrm{g}$. $\mathbf{h}$, Schematic of the working mechanism 576 of the energy harvesting and storing module. i, Photocharging and discharging curves of the battery 577 fibre. Six photovoltaic units in series under illumination are used to charge zinc-ion battery fibres. 578 The battery fibres are discharged to an external circuit at a current of $80 \mu \mathrm{A}$. 

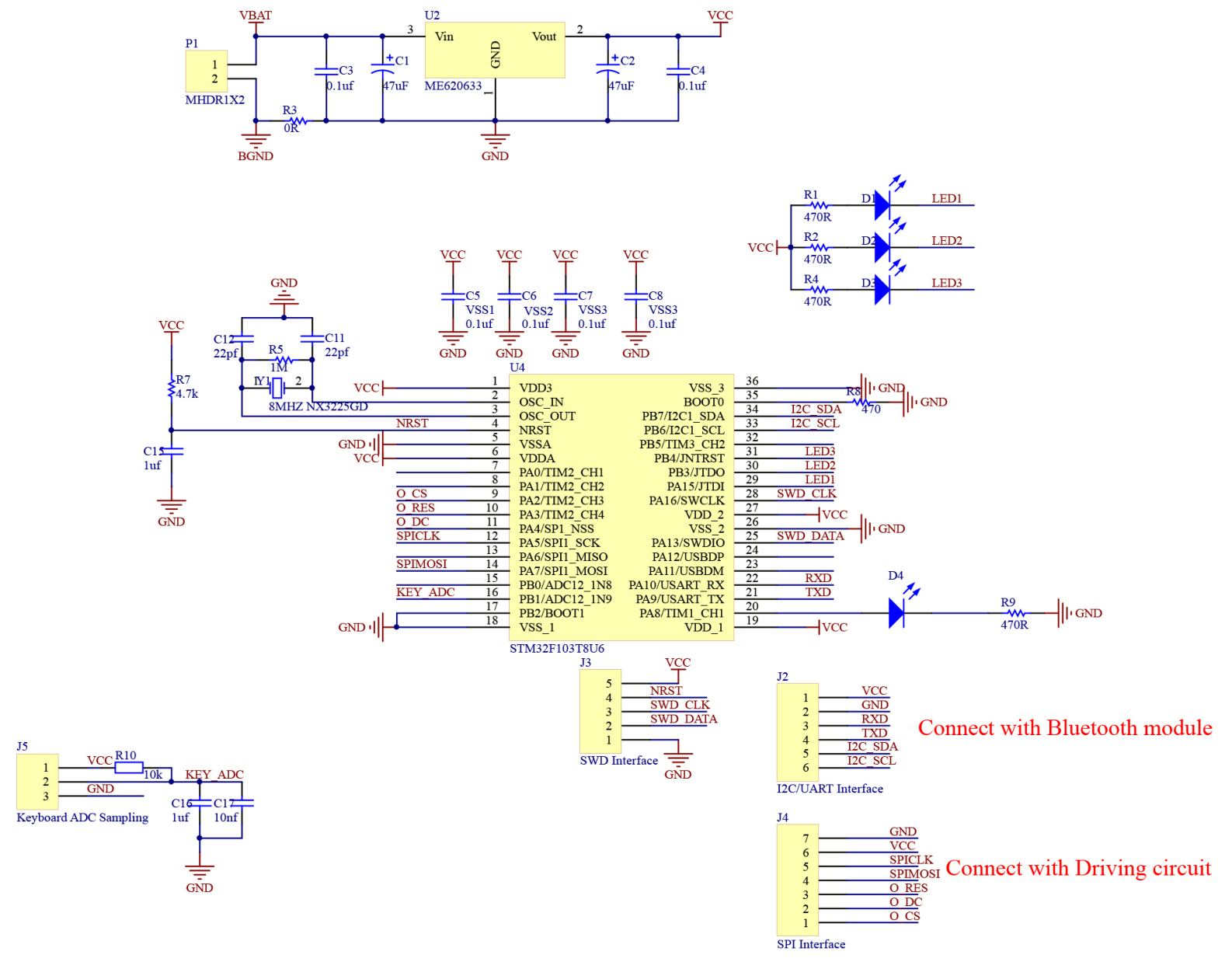

581 Extended Data Fig. 9 | Schematic diagram of the integrated textile system. 


\section{Figures}

a
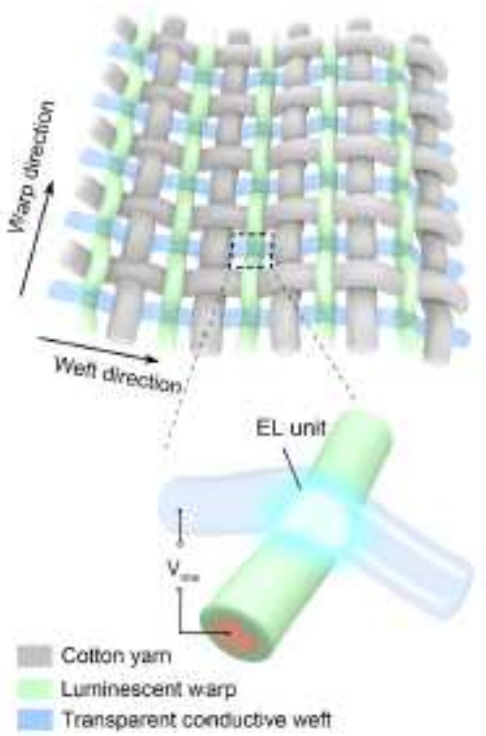

。

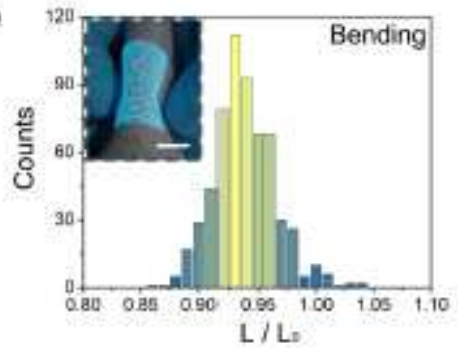

h

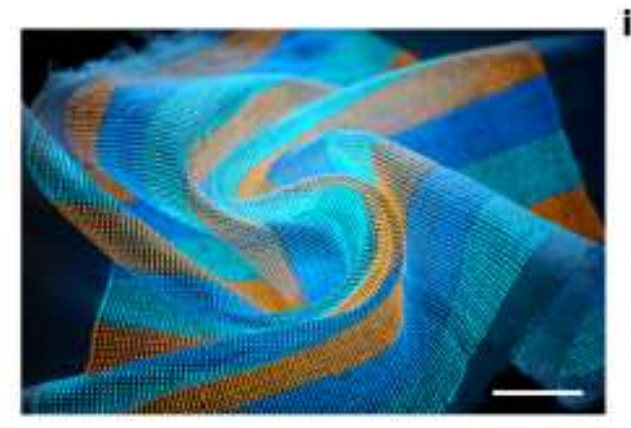

b
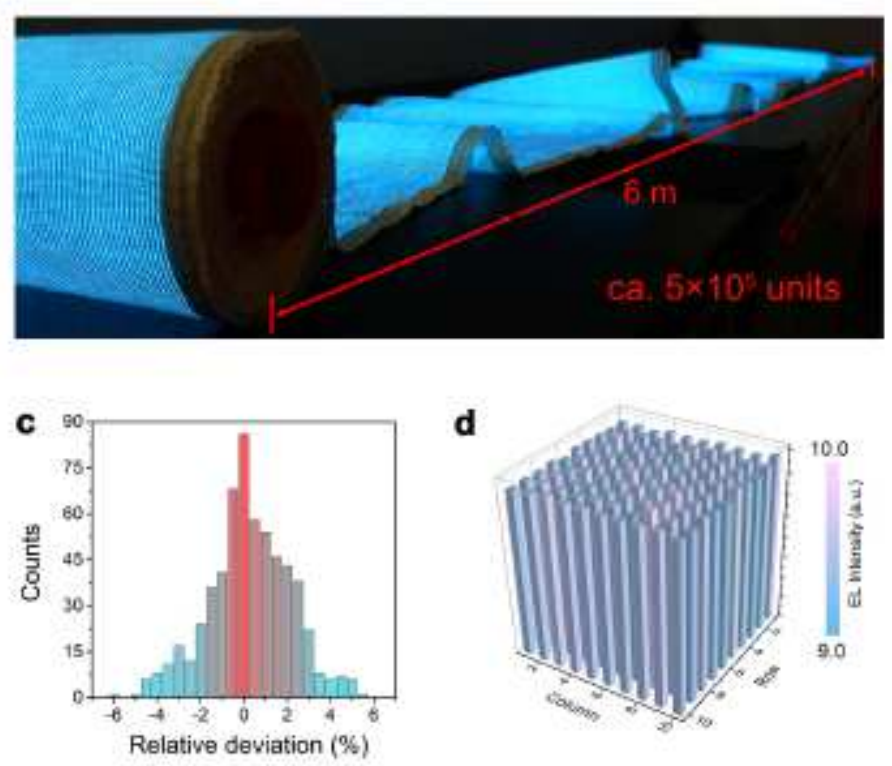

d
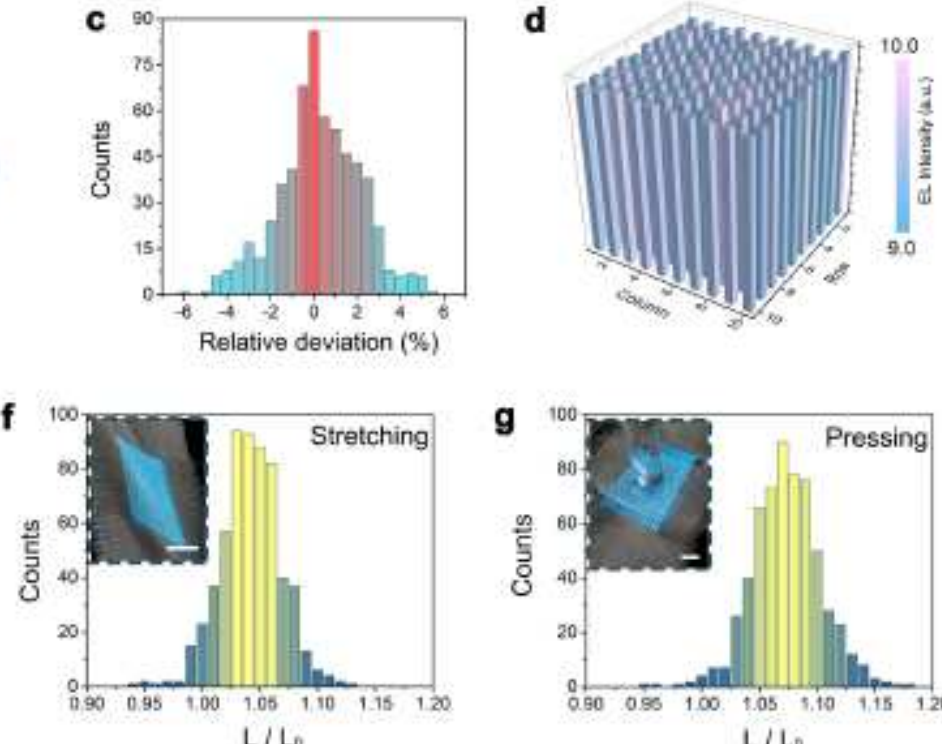

g

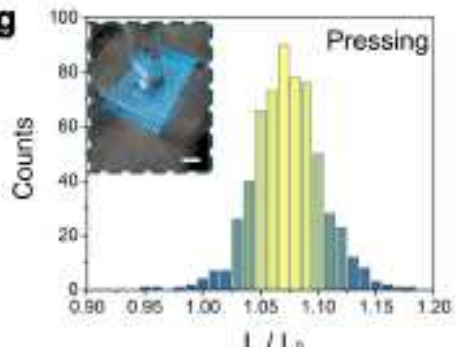

j

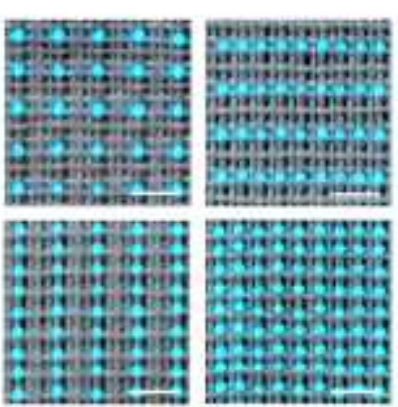

\section{Figure 1}

Structure and EL performance of the display textile. a, Schematic showing the weave diagram of the display textile. Each contacting luminescent warp and transparent conductive weft forms an EL unit (inset). Applied alternating voltage (Vrms) turns on the EL units. b, Photograph of a 6-metre-long display textile consisting of approximately $5 \times 105 \mathrm{EL}$ units. c, Statistical distribution shows the relative deviations of emission intensity for 600 EL units examined are between $-6.3 \%$ and $5.2 \%$. Relative deviation is defined as deviation of luminance for a single EL unit from the average value. $d$, Emission intensities of a $10 \times 10$ EL unit array is uniform (< 10\% 440 differences in intensity among the units). e-g, Statistical distribution shows minor (< 10\%)variations in luminance for $600 \mathrm{EL}$ units after 1,000 cycles of bending (e), stretching 
(f), and pressing (g). Insets: photographs of tested samples. Scale bars, $1 \mathrm{~cm}$. h, Photograph of a functional multicolour display textile under complex deformations, including bending and twisting. Blue and orange are achieved by doping ZnS with copper and manganese, respectively. Scale bar, $2 \mathrm{~cm}$. i, Magnified photograph of the multicolour display textile from $\mathrm{h}$ show EL units are uniformly spaced at a distance of $\sim 800 \mu \mathrm{m}$. Scale bar, $2 \mathrm{~mm}$. j, Photographs of EL units spaced at different distances obtained by changing the weaving parameters. Scale bars, $2 \mathrm{~mm}$.

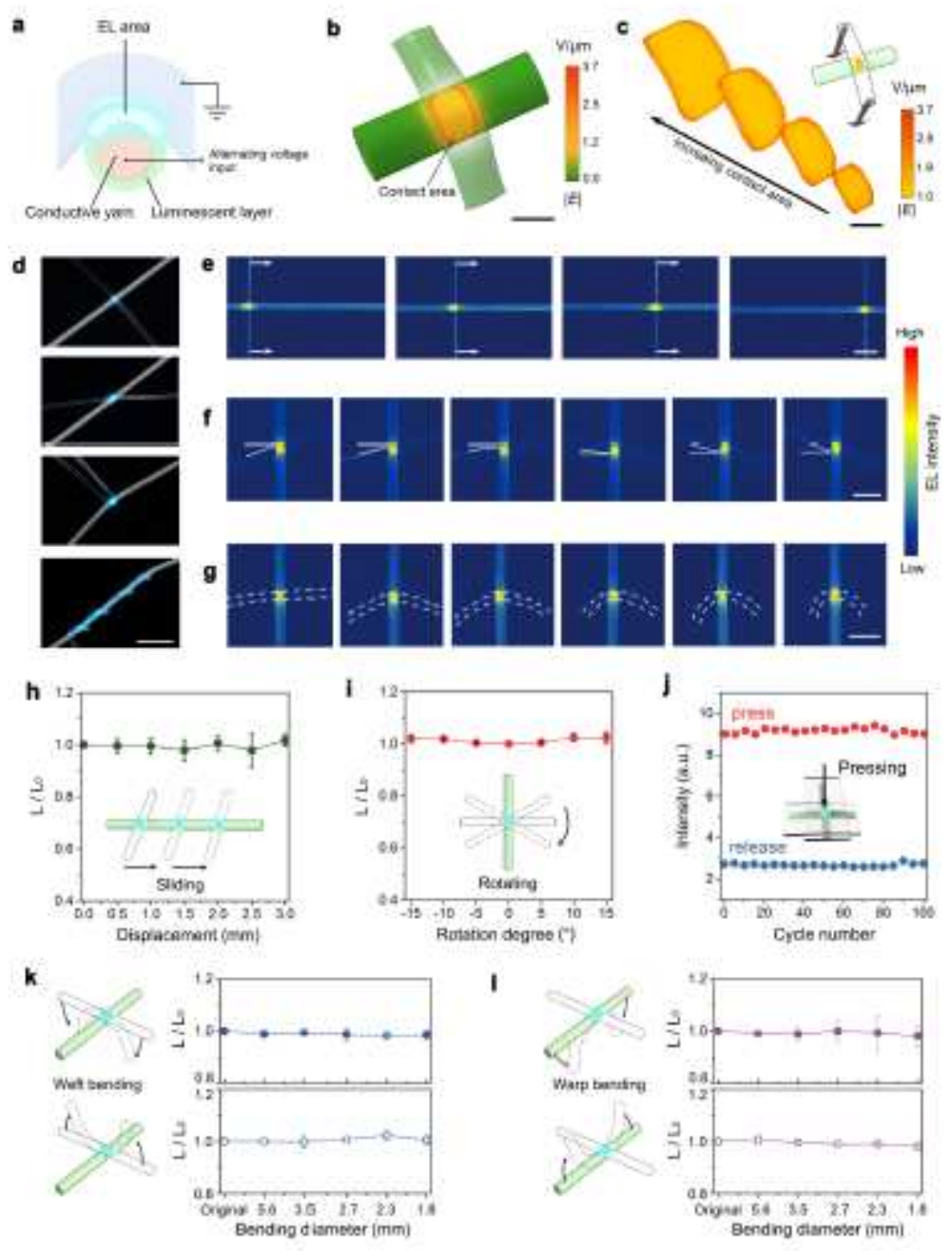

Figure 2

Characterization of EL units of the display textile. a, Schematic of an EL unit formed at the contact area between luminescent warp and transparent conductive weft. Light emission occurs when an alternating electric voltage is applied. b, c, Simulation using finite element method shows electric field distribution at the contact area in an EL unit is uniform (b) and does not change with increasing contact areas (c). $d$, Photographs show stable light emission as the transparent conductive weft contacted, leaned, twisted and knotted with the luminescent warp (top to bottom). Scale bar, $2 \mathrm{~mm}$. e-g, EL maps show brightness of EL units remained stable even when the transparent weft is slid (e), rotated (f) and bent (g) along the luminescent warp. Colour bar indicates relative EL intensity. Scale bars, $1 \mathrm{~mm}$. h-I, Luminance varied minimally when the transparent weft is moved by $3 \mathrm{~mm}$ along the luminescent warp $(\mathrm{h})$ and rotated by 
different degrees ( $i ; 0^{\circ}$ is when the weft is perpendicular to the warp), and when the EL unit is pressed and released for 100 cycles $(j)$, bent along the weft length $(k)$ and along the warp length ( $I)$ with increasing bending angles. $\mathrm{LO}$ and $\mathrm{L}$ correspond to the $\mathrm{EL}$ intensity before and after deformation, respectively. Error bars are standard deviations of the results from at least three samples.
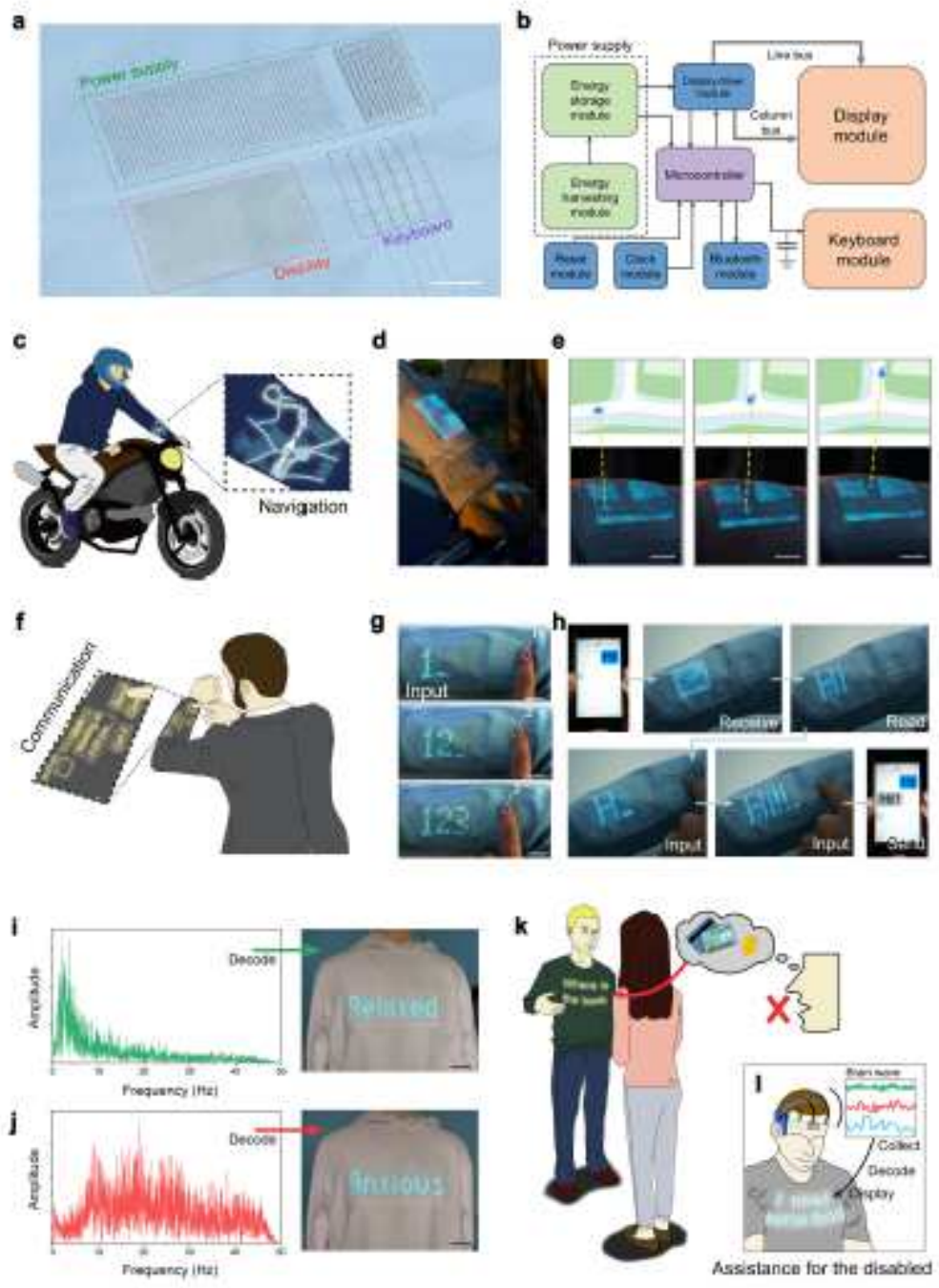

\section{Figure 3}

Application scenarios of integrated textile systems. a, Photograph of an integrated textile system consisting of display, information input (keyboard) and power supply modules. Scale bar, $2 \mathrm{~cm}$. b, System-level block diagram of the integrated textile system in a shows the modules connected to a microcontroller that is powered by solar energy harvesting and electrical energy storing modules. c, Schematic illustrating the integrated textile as a smart node for the Internet of Things to offer location services during driving. Selective illumination on the display module is achieved by scanning electrical signals from the driver circuit row by row onto the array of EL units. d, e, Real-time location at a T-junction on a sleeve, transferred through the Bluetooth and microcontroller modules, is synchronized with the location map on a smartphone. Scale bars, $1 \mathrm{~cm}$. $\mathrm{f}$, Schematic shows textiles integrated with a display and keyboard can be used as a communication platform. $\mathrm{g}$, Information is input onto clothes by pressing 
the keypress that is woven into the textile. Scale bars, $2 \mathrm{~cm}$. h, Receiving and sending messages between the integrated textile system and a smartphone. i, j, Expressions of mental states by decoding representative electroencephalogram signals. The words "Relaxed" (i) and "Anxious" (j) are displayed on clothes when the dominant brain waves are detected in the low-frequency region $(0-10 \mathrm{~Hz})$ and highfrequency region $(10-40 \mathrm{~Hz}$ ), respectively. Scale bars, $5 \mathrm{~cm}$. $\mathrm{k}$, Display textiles could in the future enable individuals with speech disorders to communicate and express themselves. I, Conceptual image showing brain waves from a disabled person are being decoded into messages that are displayed on a shirt made from an integrated textile.

\section{Supplementary Files}

This is a list of supplementary files associated with this preprint. Click to download.

- SupplementaryMovie1.mp4

- SupplementaryMovie2.mp4

- SupplementaryMovie3.mp4

- Figs1.png

- Figs2.png

- FigS3.png

- Figs4.png

- Figs5.png

- Figs6.png

- Figs7.png

- Figs8.png

- Figs9.png 